Document downloaded from:

http://hdl.handle.net/10251/136184

This paper must be cited as:

Medina, JR.; Hudspeth, RT. (1990). A review of the analyses of ocean wave groups. Coastal Engineering. 14(6):515-542. https://doi.org/10.1016/0378-3839(90)90033-S

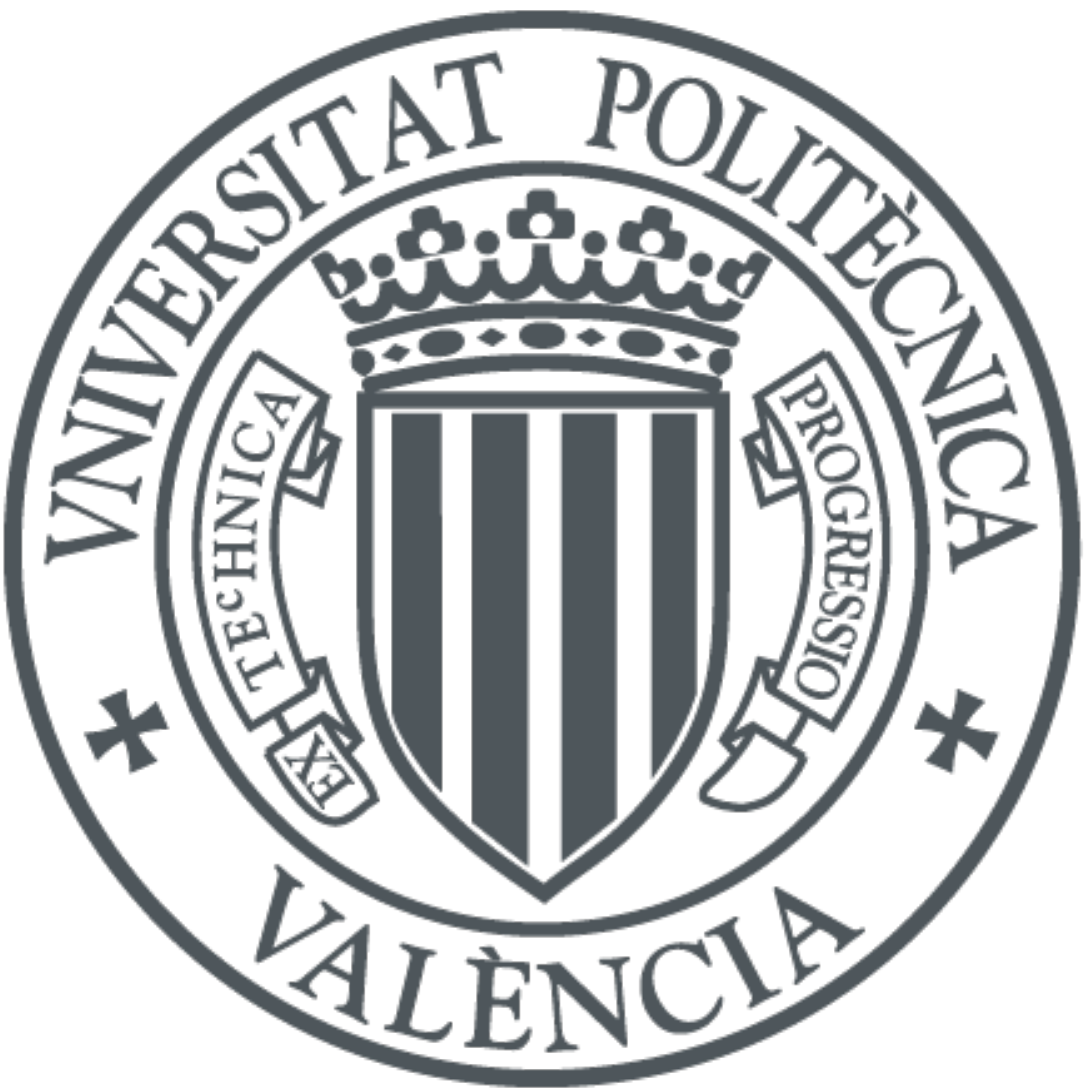

The final publication is available at

https://doi.org/10.1016/0378-3839(90)90033-S

Copyright Elsevier

Additional Information 


\title{
A REVIEW OF THE ANALYSES OF OCEAN WAVE GROUPS
}

\author{
J.R. Medina ${ }^{1}$ and R.T. Hudspeth ${ }^{2}$ \\ ${ }^{1}$ Universidad Politécnica de Valencia, Valencia, Spain \\ ${ }^{2}$ Oregon State University, Corvallis, Oregon, U.S.A.
}

\section{ABSTRACT}

The most common parameters and functions used to characterize wave groups in linear seas are reviewed and interrelated in a unified manner. A threeaxis representation of run lengths is used to characterize wave groups using exponential and Markov chain approximations. A relationship between four parameters $\left(Q_{p}, Q_{e}, \kappa^{2}=4 \rho^{2}\right)$ and the correlation coefficient between consecutive wave heights $\left(r_{H H}(1)\right)$ is demonstrated. The wave height function method is reviewed in some detail in order to relate the run length theory with envelope theories. The theoretical estimates used to demonstrate the relationships between the various parameters must be considered as only firstorder trends to parameter estimates computed from real wave data due to the statistical variability in these estimates. 


\section{NOMENCLATURE}
$A(t)$
envelope function

$A F(t)$

analytical function

$A_{1}(\tau)$

envelope of the $i^{\text {th }}$ function

$a_{i}\left[=A\left(t_{i}\right)\right] \quad$ discrete value of envelope function

B

rectangular spectrum bandwidth

$\mathrm{C}$ constant of proportionality in Eq. (47)

\section{$E[\cdot]$}

expected value

E

complete elliptical integral of the second kind

f

frequency

$f_{m}$

frequency of the $\mathrm{m}^{\text {th }}$ wave component

$f_{N}(=1 / 2 \Delta T)$

Nyquist frequency

GF

Groupiness Factor (SIWEH)

$H(t)$

wave height function

$\mathrm{H}_{\text {med }}$

median wave height

$\mathrm{H}_{\mathrm{rms}}$

root-mean-square wave height

$\mathrm{H}_{\mathrm{s}}$

significant wave height

$\overline{\mathrm{H}}$

mean wave height

$\mathrm{H}_{\mathrm{i}}$ Not

discrete wave height

h

threshold leve1

$\mathrm{I}_{0}(\cdot)$

modified Bessel function of the first kind of order zero

$j(=\sqrt{-1})$

imaginary unit number

K

complete elliptical integral of the first kind

$\mathrm{k}\left(=\mathrm{T}_{01} / \nu=\mathrm{T}_{02} / \delta\right)$ parameter

${ }_{1} L_{h}(m)$

length of the $m^{\text {th }}$ run of high waves above threshold level, $h$

${ }_{2} L_{h}(m)$

length of the $\mathrm{m}^{\text {th }}$ total run of waves above threshold level, $h$ 


\begin{tabular}{|c|c|}
\hline${ }_{1} \ell_{h}^{\prime}\left[\ell_{h}^{\prime \prime}\right]$ & $\begin{array}{l}\text { length of a run of high waves computed using the envelope and } \\
\text { normalized by } \mathrm{T}_{01}\left[\text { by } \mathrm{T}_{02}\right]\end{array}$ \\
\hline${ }_{2} \ell_{h}^{\prime}\left[{ }_{2} \ell_{h}^{\prime \prime}\right]$ & $\begin{array}{l}\text { length of a total run of waves computed using the envelope and } \\
\text { normalized by } T_{01} \text { [by } T_{02} \text { ] }\end{array}$ \\
\hline LVTS & Local Variance Time Series \\
\hline$m_{n}$ & $\mathrm{n}^{\text {th }}$ spectral moment \\
\hline$P(\cdot)$ & cumulative distribution function (c.d.f.) \\
\hline $\mathrm{p}(\cdot)$ & probability density function (p.d.f.) \\
\hline$p(\cdot, \cdot)$ & joint probability density function \\
\hline$Q_{e}$ & dimensionless spectral peakedness parameter defined by Eq. (41) \\
\hline$a_{p}$ & dimensionless spectral peakedness parameter defined by Eq. (1) \\
\hline$q(\cdot)$ & marginal probability density function \\
\hline $\mathrm{R}_{1}(\tau)$ & autocorrelation function of $i^{\text {th }}$ variable \\
\hline $\mathrm{R}_{\mathrm{m}}$ & amplitude of the $\mathrm{m}^{\text {th }}$ wave component \\
\hline$r_{H H}(m)$ & $\begin{array}{l}\text { correlation coefficient between successive wave heights with a } \\
\mathrm{lag}(\mathrm{m})\end{array}$ \\
\hline$r_{T T}(m)$ & $\begin{array}{l}\text { correlation coefficient between successive wave periods with } \\
\text { lag m }\end{array}$ \\
\hline$r_{H T}(m)$ & $\begin{array}{l}\text { correlation coefficient between successive wave heights and } \\
\text { periods with } 1 \mathrm{ag} \mathrm{m}\end{array}$ \\
\hline $\operatorname{SIWEH}(t)$ & Smoothed Instantaneous Wave Energy History \\
\hline$S_{i}(f)$ & one-sided variance spectrum of $i^{\text {th }}$ variable \\
\hline $\mathrm{T}_{01}\left(=\mathrm{m}_{0} / \mathrm{m}_{1}\right)$ & mean orbital period \\
\hline $\mathrm{T}_{02}\left(=\sqrt{\mathrm{m}_{0} / \mathrm{m}_{2}}\right)$ & mean zero upcrossing period. \\
\hline $\mathrm{T}_{\text {SHH }}$ & zero upcrossing period of SIWEH \\
\hline$T_{p}\left(=f_{p}^{-1}\right)$ & period of spectral peak \\
\hline & emporal spectral peakedness pa \\
\hline
\end{tabular}




\begin{tabular}{|c|c|}
\hline$t$ & time \\
\hline$\mu_{3(4)}$ & cosine (sine) transform of $S_{\eta}(f)$ \\
\hline$\gamma$ & peak enhancement factor for Goda-JONSWAP spectrum \\
\hline$\Gamma_{\eta}(f)$ & envelope spectral density function (unit variance) \\
\hline$\Delta \mathrm{T}$ & sampling time interval of squared-wave height function \\
\hline$\delta^{2}$ & $\begin{array}{l}\text { Vanmarcke (1972) dimensionless spectral bandwidth parameter } \\
\text { water surface elevation [Hilbert Transform] }\end{array}$ \\
\hline$\theta_{\mathrm{m}}$ & random phase angle of the $\mathrm{m}^{\text {th }}$ wave component \\
\hline$\theta(\mathrm{t})+\theta$ & instantaneous phase angle \\
\hline$\kappa$ & correlation parameter used in Eq. (18) \\
\hline$\nu$ & $\begin{array}{l}\text { Longuet-Higgins (1957) dimensionless spectral bandwidth } \\
\text { parameter }\end{array}$ \\
\hline$\rho$ & correlation parameter used in Eqs. (2 \& 3) \\
\hline$\sigma^{2}(\cdot)$ & variance \\
\hline${ }_{1} \tau_{\mathrm{h}}$ & exceedance time interval of envelope above threshold 1evel $\mathrm{h} / 2$ \\
\hline $2^{T} \mathrm{~h}$ & $\begin{array}{l}\text { time interval between consecutive upcrossings of envelope above } \\
\text { threshold level } \mathrm{h} / 2\end{array}$ \\
\hline$\Omega(t)$ & local frequency function \\
\hline$(\bar{\cdot})$ & average value of $(\cdot)$ \\
\hline$(\hat{\bullet})$ & Hilbert Transform of $(\cdot)$ \\
\hline
\end{tabular}




\section{INTRODUCTION}

The tendency of ocean waves to appear in groups is receiving increased attention by coastal and ocean engineers. There is a general agreement that wave grouping characteristics affect the stability of some types of maritime structures. Table 1 is a list of references in which wave groups were used to analyze various coastal phenomena and engineering problems.

\section{[INSERT TABLE 1]}

In spite of the evidence that wave groups are important in a variety of coastal and ocean applications, our ability to incorporate the effects of wave groups into current design methods is limited. The difficulty of including the effects of wave groups into engineering design is due in part to the variety of theories and parameters used to characterize wave groups. Table 2 summarizes the parameters and functions from various methodologies that are used to analyze and to characterize wave groups. It is obvious from Table 2 that the variety of parameters and functions from the various methodologies makes it difficult to incorporate the wave group concept into engineering design in a consistent manner. Here we attempt to identify the similarities and relationships among the various parameters, functions, and methodologies most commonly used to analyze wave groups. $\cdots$

\section{[INSERT TABLE 2]}

A previous review by Rye (1982) of the different wave group parameters and methodologies led to a conclusion that wave groups measured from field data compared quite well with those obtained from numerical simulations derived from linear algorithms. The validity of the linear hypothesis was also obtained by Goda (1983) and Elgar et a1. (1984, 1985) from their analyses of real ocean waves measured in water depths greater than 10 meters. Field 
observations from Battjes and Vledder (1984) also support the linear hypothesis. Therefore, nonlinear wave-wave interaction models will not be included in this comparison of methods used to analyze wave groups in linear, random seas.

The parameters and functions 1 isted in Table 2 that are used in the various methodologies will first be identified and interrelated where possible. Next, three methods of analyses of wave groups which incorporate most of these parameters and functions will be reviewed in some detail. These three methods of analyses are: 1) wave height function; 2) three-axis representation of run lengths; and 3) correlation coefficient between successive wave heights.

\section{REVIEW OF WAVE GROUP METHODOLOGIES}

Four decades ago, Tucker (1950) identified the presence of waves in the surf zone with periods between 1 to 5 minutes and suggested that these long waves were generated by wave groups. Although Tucker (1950) noted the importance to engineering design from these wave groups in the analysis of harbor resonance, the real importance of wave grouping did not arise until the seminal study by Goda (1970).

In this seminal study, Goda (1970) used linear numerical simulations to demonstrate that ocean waves in a random field are not completely randomly distributed. Instead, real ocean waves demonstrate a tendency to appear in groups in a manner that depends on the peakedness of the spectrum. He introduced the concepts of a length of a run of high waves and of a length of a total run of waves as well as the peakedness parameter, $Q_{p}$. The methods and parameters introduced by Goda (1970) have been the most widely used in the succeeding studies listed in Table 2 . 


\section{Run Lengths (Goda, 1970)}

The length of a run of high waves, ${ }_{1} L_{h}$, is defined as the number of consecutive wave heights that are higher than a specified threshold value (e.g., $h=H_{r m s}, H_{s}, \vec{H}, H_{\text {med }}, \ldots$ ). The length of a total run of waves, ${ }_{2} L_{h}$, is the total number of wave heights that occur between the time of the first exceedance above the specified threshold value, $h$, and the time of the first re-exceedance above the same specified threshold value.

\section{[INSERT FIG. 1 HERE]}

Figure 1 illustrates the procedure used to determine the run lengths from a sequence of wave heights. In Fig. $1, h=$ the specified threshold wave height; ${ }_{1} L_{h}(m)=$ the $m^{\text {th }}$ length of a run of high waves; and ${ }_{2} L_{h}(m)=$ the $m^{\text {th }}$ length of a total run of waves.

Goda (1970) derived a probability density function (p.d.f.) for ${ }_{1} L_{h}$ and ${ }_{2} L_{h}$ for uncorrelated wave heights. However, linear numerical simulations by Goda (1970) showed that if $\eta(t)$ was a realization of an ergodic Gaussian stochastic process, the corresponding p.d.f. of $\mathrm{L}_{h}$ and ${ }_{2} \mathrm{~L}_{h}$ departed significantly from the uncorrelated case as a function of the spectral shape.

High values of ${ }_{1} L_{h}$ and ${ }_{2} L_{h}$ are associated with larger wave groups. Therefore, parameters like the average run lengths of ${ }_{1} \bar{L}_{h}$ and ${ }_{2} \bar{L}_{h}$, and the sample p.d.f., $p\left({ }_{1} L_{h}\right)$ and $p\left({ }_{2} L_{h}\right)$, have been widely used to characterize wave groups in ocean wave records. Also a variety of threshold levels, h, have been used $\left(h=H_{s}, H_{\text {med }}, \bar{H}, \ldots\right)$.

The numerical experiments of Goda (1970) indicated that the characteristics of wave groups in irregular wave trains were correlated with spectral peakedness. The dimensionless spectral peakedness parameter introduced by Goda (1970) was 


$$
Q_{p}=\frac{2 \int_{0}^{\infty} f S_{\eta}^{2}(f) d f}{\left[\int_{0}^{\infty} S_{\eta}(f) d f\right]^{2}}
$$

The statistical properties of the run lengths introduced by Goda (1970) were further refined by Kimura (1980).

\section{Markov Chain Hypothesis (Kimura, 1980)}

To analyze the statistical properties of the run lengths, Kimura (1980) introduced a Markov chain hypothesis for a sequence of wave heights. He estimated a p.d.f. for ${ }_{1} L_{h}$ and ${ }_{2} L_{h}$ as a function of a single correlation parameter, $\rho$. The joint p.d.f., $p\left(H_{1}, H_{2}\right)$, for two successive wave heights, $H_{1}=H_{1}$ and $H_{2}=H_{i+1}$, is given by

$$
p\left(H_{1}, H_{2}\right)=\frac{4 H_{1} H_{2}}{\left(1-4 \rho^{2}\right) H_{r m s}^{4}} \exp \left[-\left[1 /\left(1-4 \rho^{2}\right)\right]\left[\frac{H_{1}^{2}+H_{2}^{2}}{H_{r m s}^{2}}\right]\right] I_{0} \cdot\left[\frac{4 H_{1} H_{2} \rho}{\left(1-4 \rho^{2}\right) H_{r m s}^{2}}\right]
$$

where $\rho=$ the correlation parameter, $H_{r m s}=$ the root-mean-square value of wave heights, and $I_{0}(\cdot)=$ the modified Bessel function of the first kind of order zero. The correlation coefficient between consecutive wave heights is given by

$r_{H H}(1)=\frac{E-\left(1-4 \rho^{2}\right) K / 2-\pi / 4}{1-\pi / 4}$

where $\mathrm{K}$ and $\mathrm{E}=$ the complete elliptic integrals of the first and second kinds, respective1y, with parameter (2p) [cf. Abramowitz and Stegun, 1968, p. 590].

The probability $P_{1}$ that $H_{2}$ does not exceed $h$ when $H_{1}$ is below the threshold height, $h$; and the probability $\mathrm{P}_{2}$ that $\mathrm{H}_{2}$ does exceed the threshold level $h$ when $H_{1}$ also exceeds $h$, may be defined as follows:

$$
P_{1}=\int_{0}^{h} \int_{0}^{h} p\left(H_{1}, H_{2}\right) d H_{1} d H_{2} / \int_{0}^{h} q\left(H_{1}\right) d H_{1}
$$




$$
P_{2}=\int_{h}^{\infty} \int_{h}^{\infty} p\left(H_{1}, H_{2}\right) d H_{1} d H_{2} / \int_{h}^{\infty} q\left(H_{1}\right) d H_{1}
$$

where $q(H)=$ a marginal p.d.f. given by the Rayleigh distribution. Kimura (1980) gives the p.d.f.'s and expected values for ${ }_{1} L_{h}$ and ${ }_{2} L_{h}$ as

$$
\begin{aligned}
& \left.p\left({ }_{1} L\right)=P_{2}{ }_{1} L-1\right)\left(1-P_{2}\right) \\
& E\left({ }_{1} L\right)=1 /\left(1-P_{2}\right) \\
& p\left({ }_{2} L\right)=\frac{\left(1-P_{1}\right)\left(1-P_{2}\right)}{\left(P_{1}-P_{2}\right)}\left(P_{1}{ }^{2} 2^{L-1)}-P_{2}{ }_{2}{ }^{L-1)}\right) \\
& E\left({ }_{2} L\right)=\frac{1}{\left(1-P_{1}\right)}+\frac{1}{\left(1-P_{2}\right)}
\end{aligned}
$$

where ${ }_{i} L={ }_{i} L_{h}$ for notational convenience.

Because of the relationship between the correlation parameter, $\rho$, and the correlation coefficient between consecutive wave heights, $r_{H H}(1)$, given by Eq. (3); $\mathrm{p}\left({ }_{1} \mathrm{~L}\right)$ and $\mathrm{p}\left({ }_{2} \mathrm{~L}\right)$ depend on only one single parameter; viz., $r_{H H}(1)$.

Goda (1983) found $r_{H H}(1)$ to be an excellent parameter to describe the run lengths from an analysis of long-traveled swell waves. He also found that the long-traveled swell data agreed quite well with the approximations in the Kimura theory. Battjes and Vledder (1984) observed that the distribution of lengths of runs exceeding $h=H_{1 / 3}$ in records from the North Sea also agreed quite well with the Kimura theory. They suggested using the parameter $\kappa^{2}=(2 \rho)^{2}$ derived from the variance spectrum $S_{\eta}(f)$ to characterize the wave groups.

Earlier, Rye (1974) had identified the parameter $r_{H H}(\cdot)$ as a parameter useful to analyze wave groups. 


\section{Correlation Coefficient for Succeeding Waves (Rye, 1974)}

Rye (1974) identified the presence of wave groups in real ocean wave records using correlation coefficients computed from time series of wave heights and wave periods. However, only $r_{H H}(1)$ has received significant attention. The correlation coefficient for successive wave heights is given by

$$
r_{H H}(m)=\frac{1}{r_{H H}(0)} \frac{1}{(M-m)} \sum_{i=1}^{(M-m)}\left(H_{i}-\bar{H}\right)\left(H_{i+m}-\bar{H}\right) ;
$$

for wave periods by

$$
r_{T T}(m)=\frac{1}{r_{T T}(0)} \frac{1}{(M-m)} \sum_{i=1}^{(M-m)}\left(T_{i}-\bar{T}\right)\left(T_{i+m}-\bar{T}\right) ;
$$

and for both wave heights and wave periods by

$$
r_{H T}(m)=\frac{1}{\sigma(T) \sigma(H)} \frac{1}{(M-m)} \sum_{i=1}^{(M-m)}\left(H_{i}-\bar{H}\right)\left(T_{i+m}-\bar{T}\right) .
$$

where $\left(H_{i}, T_{i}\right)=$ the wave height and the wave period, respectively, of the $i^{\text {th }}$ wave in a record; and $M=$ total number of waves in the series. The variances are given by $r_{H H}(0)=\sigma^{2}(H)$ and $r_{T T}(0)=\sigma^{2}(T)$.

[INSERT FIG. 2 HERE]

- The correlation coefficient between succeeding wave heights, $r_{H H}(m)$, can be related to the autocorrelation function of the envelope or the wave height function. From the relationships between $H_{i}, A(t)$ and $H(t)$ illustrated in Fig. 2, we may deduce that

$$
R_{A}(m \bar{T})=R_{H}(m \bar{T}) \doteq r_{H H}(m)
$$

where $\bar{T}=$ a characteristic mean period; $R_{A}(\tau)=$ the autocorrelation function of the wave envelope, $A(t)$; and $R_{H}(\tau)=$ the autocorrelation function of the wave height function, $H(t)$. 
The envelope function, $A(t)$, had been identified earlier by Rice (1954) in his analysis of random noise.

\section{Envelope and Wave Height Function (Rice, 1954)}

Nolte and Hsu (1972) observed that consecutive high waves in a group of waves can excite extreme forces in the mooring lines of large floating vessels. Using the analysis of groups based on the envelope concept (vide Fig. 2), they defined the average time duration for groups above a threshold level $\mathrm{h}$ as a basic parameter which controls the grouping characteristics.

Assuming a narrow-banded process and the results given by Rice (1954), Nolte and Hsu (1972) calculated the average duration time of excursion above a threshold level, $a=h / 2$ from

$$
{ }_{1} \bar{t}_{\mathrm{h}} \doteq \frac{\mathrm{H}_{\mathrm{s}} / \mathrm{h}}{2 \sqrt{2 \pi}} \mathrm{k}
$$

where

$$
k^{2}=\frac{1}{f_{02}^{2}-\bar{f}^{2}}
$$

where $f_{02}^{2}=m_{2} / m_{0} ; \bar{f}=m_{1} / m_{0} ; m_{n}=n^{\text {th }}$ spectral moment; and $H_{s}=$ the significant wave height.

Equation (14) may also be expressed in terms of dimensionless spectral bandwidth parameters according to

$$
\begin{aligned}
& { }_{1} \bar{\ell}_{\mathrm{h}}^{\prime}=\frac{{ }_{1} \bar{\tau}_{\mathrm{h}}}{T_{01}}=\frac{\mathrm{H}_{\mathrm{s}} / \mathrm{h}}{2 \nu \sqrt{2 \pi}}=\frac{(1 / \nu)\left(\sqrt{8 \mathrm{~m}_{0}} / \mathrm{h}\right)}{2 \sqrt{\pi}} \\
& { }_{1} \bar{\ell}_{\mathrm{h}}^{\prime \prime}=\frac{{ }_{1} \bar{\tau}_{\mathrm{h}}}{\mathrm{T}_{02}}=\frac{\mathrm{H}_{\mathrm{s}} / \mathrm{h}}{2 \delta \sqrt{2 \pi}}=\frac{(1 / \delta)\left(\sqrt{8 \mathrm{~m}_{0}} / \mathrm{h}\right)}{2 \sqrt{\pi}}
\end{aligned}
$$


where $\nu^{2}=\left(m_{0} m_{2}-m_{1}^{2}\right) / m_{1}^{2}$ is the dimensionless spectral bandwidth parameter defined by Longuet-Higgins $(1957,1984) ; \delta^{2}=1-\left(m_{1}^{2} / m_{0} m_{2}\right)$ is the dimensionless spectral bandwidth parameter defined by Vanmarcke $(1972,1983) ; \mathrm{T}_{01}=\mathrm{m}_{0} / \mathrm{m}_{1}$; $T_{02}=\sqrt{m_{0} / m_{2}}$ and $H_{s}=4 \sqrt{m_{0}}$.

Note that both ${ }_{1} \bar{\ell}_{\mathrm{h}}^{\prime}$ and ${ }_{1} \bar{\ell}_{\mathrm{h}}^{\prime \prime}$ can be considered as variables analogous to the mean length of a run of high waves, ${ }_{1} \bar{L}_{h}$. In addition, the parameter $k$, in Eq. (15), may be expressed as $k=T_{01} / \nu=T_{02} / \delta$.

Battjes and Vledder (1984) and Longuet-Higginș (1984) have identified similarities between the Kimura theory and the statistical properties of the envelope function given by Rice (1954). The joint p.d.f. between successive values of the envelope function, $p\left(a_{1}, a_{2}\right)$ is given by Rice (1954) as

$$
p\left(a_{1}, a_{2}\right)=\frac{a_{1} a_{2}}{m_{0}^{2}\left(1-\kappa^{2}\right)} \exp \left[-\frac{\left(a_{1}^{2}+a_{2}^{2}\right)}{2 m_{0}\left(1-\kappa^{2}\right)}\right] I_{0}\left(\frac{\kappa}{1-\kappa^{2}} \frac{a_{1} a_{2}}{m_{0}}\right)
$$

where $a_{1}=A\left(t_{1}\right)$ and $a_{2}=A\left(t_{1}+r\right)$, and

$$
\begin{aligned}
& \kappa=\left(\mu_{3}^{2}+\mu_{4}^{2}\right)^{1 / 2} / \mathrm{m}_{0} \\
& \mu_{3}=\int_{0}^{\infty} \mathrm{S}_{\eta}(f) \cos \left[2 \pi\left(f-f_{01}\right) \tau\right] \mathrm{df} \\
& \mu_{4}=\int_{0}^{\infty} \mathrm{S}_{\eta}(f) \sin \left[2 \pi\left(f-f_{01}\right) \tau\right] \mathrm{df}
\end{aligned}
$$

and $I_{0}=$ the modified Bessel function of the first kind of order zero defined by (cf. Abramowitz and Stegun, 1968, p. 376)

$$
I_{0}(z)=\frac{1}{\pi} \int_{0}^{\pi} \exp (z \cos \theta) d \theta
$$

Longuet-Higgins (1984) assumed that $\tau=\mathrm{m}_{0} / \mathrm{m}_{1}=\mathrm{T}_{01}$ and defined a corre1ation coefficient between successive wave heights by 


$$
R_{A}\left(T_{01}\right)=M_{11} /\left(M_{20} M_{02}\right)^{1 / 2}
$$

where

$$
M_{p q}=\int_{0}^{\infty} \int_{0}^{\infty}\left(a_{1}-\bar{a}\right)^{p}\left(a_{2}-\bar{a}\right)^{q} \quad p\left(a_{1}, a_{2}\right) d a_{1} d a_{2}
$$

This correlation coefficient may be computed by

$$
R_{A}\left(T_{01}\right)=\frac{E-\left(1-\kappa^{2}\right) K / 2-\pi / 4}{1-\pi / 4}
$$

where $K$ and $E=$ the complete elliptic integrals of the first and second kind, respectively, with parameter $\kappa$. Equation (25) is identical to Eq. (3) from Kimura (1980) for $\kappa=2 \rho$.

For narrow-banded processes, Longuet-Higgins (1984) found that

$$
\kappa^{2} \doteq 1-4 \pi^{2} \nu^{2}
$$

while Eq. (25) has the approximation

$$
\mathrm{R}_{\mathrm{A}}\left(\mathrm{T}_{01}\right) \doteq \kappa^{2} \quad ; 0 \leq \kappa^{2} \leq 1
$$

Defining a wave height function as $H(t)=2 A(t)$ and a threshold level as $h=2 a$, then a change of variables given by

$$
p\left(h_{1}, h_{2}\right) d h_{1} d h_{2}=p\left(a_{1}, a_{2}\right) d a_{1} d a_{2}
$$

yields

$$
\begin{aligned}
& p\left(h_{1}, h_{2}\right)=\frac{1}{4} p\left(a_{1}, a_{2}\right) \\
& p\left(h_{1}, h_{2}\right)=\frac{h_{1} h_{2}}{16 m_{0}^{2}\left(1-\kappa^{2}\right)} \exp \left[-\frac{\left(h_{1}^{2}+h_{2}^{2}\right)}{8 m_{0}\left(1-\kappa^{2}\right)}\right] I_{0}\left(\frac{\kappa}{1-\kappa^{2}} \frac{h_{1} h_{2}}{4 m_{0}}\right)
\end{aligned}
$$

Equation (30) is equal to Eq. (2) derived by Kimura when $\kappa=2 \rho$, and $\mathrm{H}_{\text {rms }}=$ $\sqrt{8 \mathrm{~m}_{0}}$. 
Defining ${ }_{1} l_{h}$ and ${ }_{2} l_{h}$ as the length of a run of high waves and the length of a total run of waves computed from the envelope function at a level $a=$. $h / 2$, Longuet-Higgins (1984) found the following estimates for $E\left[l_{1} \ell_{h}\right]$ and $E\left[{ }_{2} \ell_{h}\right]$ :

$$
E\left[{ }_{1} \ell_{2 \mathrm{a}}\right] \doteq(2 \pi)^{-1 / 2}\left[\left(1+\nu^{2}\right)^{1 / 2} / \nu\right] /\left(\mathrm{a} / \sqrt{\mathrm{m}_{0}}\right)
$$

which can be rewritten as

$$
E\left[l_{1} l_{h}\right] \doteq \frac{1 / \delta}{2 \sqrt{\pi}}\left(\sqrt{8 m_{0}} / h\right)
$$

and

$$
E\left[{ }_{2} \ell_{2 a}\right] \doteq(2 \pi)^{-1 / 2}\left[\left(1+\nu^{2}\right)^{1 / 2} / \nu\right] \exp \left[a^{2} / 2 m_{0}\right] /\left(a / \sqrt{m_{0}}\right)
$$

which reduces to

$$
E\left[{ }_{2} \ell_{h}\right]=E\left[l_{1} \ell_{h}\right] \exp \left[h^{2} / 8 m_{0}\right]
$$

since $\left[\left(1+\nu^{2}\right)^{1 / 2} / \nu\right]=1 / \delta$ and $h=2 \mathrm{a}$.

Note the similarity between $E\left[{ }_{1} \ell_{h}\right]$ in $E q$. (32) and ${ }_{1} \bar{\ell}_{h}^{\prime \prime}$ in Eq. (17). If $T_{01}$ is defined to be the mean wave period, then Eq. (32) can be transformed to Eq. (16) instead of Eq. (17).

The square of the envelope or wave height function may be related to other methods to analyze wave groups.

\section{$\eta^{2}(t)$ Filters and $\mathrm{H}^{2}(\mathrm{t})$}

Medina and Hudspeth (1987) and Hudspeth and Medina (1988) identified the following similarities between the Smoothed Instantaneous Wave Energy History (SIWEH) (Funke and Mansard, 1979); the Local Variance Time Series (LVTS) (Thompson and Seelig, 1984); and the squared wave height function $\left(H^{2}(t)\right.$ ) defined from the envelope of the record:

$$
\operatorname{SIWEH}(t) \doteq \operatorname{LVTS}(t) \doteq H^{2}(t) / 8=A^{2}(t) / 2
$$


Hudspeth and Medina (1988) observed that the squared wave height function, $H^{2}(t)$, defined on the basis of a time series and its Hilbert transform isolates exactly the low frequency components of the squared water surface elevation, $\eta^{2}(t)$, for a linear stochastic process. In contrast, the SIWEH requires an arbitrary low-pass filter in order to isolate the low frequency contributions. They also found that for linear waves the expected value of the Groupiness Factor (GF) as defined by Funke and Mansard should be approximately equal to unity independent of the shape of the spectrum. This implies that the Groupiness Factor is not an appropriate parameter to characterize wave groupiness.

Rye (1982) introduced a more appropriate parameter which was later verified by Goda (1983). This parameter was the mean zero-upcrossing period of SIWEH, $\overline{\mathrm{T}}_{\text {SHH}}$, defined by

$$
\overline{\mathrm{T}}_{S H H}=\frac{1}{T_{p}} \frac{1}{\bar{I}} \sum_{i=1}^{I}\left(T_{S H H}\right)_{i}
$$

in which $I$ = the total number of zero upcrossings of the mean level in the SIWEH; and $T_{p}=1 / f_{p}=$ the period of the peak frequency of the spectrum.

The relationship between SIWEH(t) and $H^{2}(t)$ given in Eq. (35) implies that $\overline{\mathrm{T}}_{\text {SHH }}$ may be related to the mean length of the total run of waves at the threshold level $h=H_{\text {rms }}$. From Fig. 2 and Eq. (36) we find, approximately

$$
\overline{\mathrm{T}}_{\text {SHH }} \cong \frac{{ }^{2} \bar{T}_{H_{\text {rms }}}}{T_{p}}={ }_{2} \bar{\ell}_{r_{\text {rms }}}\left(\frac{T_{01}}{\bar{T}_{p}}\right)
$$

where ${ }_{2} \vec{\tau}_{H_{\text {rms }}}=$ the mean duration of the total run of waves; and ${ }_{2} \ell_{H_{\text {rms }}}=$ the length of the total run of waves using the envelope or wave height function. 
Rice (1954), Bendat and Piersol (1986), and Medina and Hudspeth (1987) give the following approximations for the spectra of $H(t)$ and $H^{2}(t)$ :

$$
S_{H}(f) \doteq(8-2 \pi) m_{0} \Gamma_{\eta}(f) ; S_{H 2}(f) \doteq 64 m_{0}^{2} \Gamma_{\eta}(f)
$$

where the envelope spectral density function (unit variance) is defined by

$$
\Gamma_{\eta}(f)=\frac{2}{m_{0}^{2}} \int_{0}^{\infty} S_{\eta}(x+f) S_{\eta}(x) d x
$$

where $\sigma^{2}[H(t)]=(8-2 \pi) m_{0}$ and $\sigma^{2}\left[H^{2}(t)\right]=64 m_{0}^{2}$.

Medina and Hudspeth (1987) demonstrated that the envelope spectral density function (unit variance), $\Gamma_{\eta}(f)$, given in Eq. (39) is usually a monotonically decreasing function with a maximum value at $f=0$. This maximum value is closely related to the grouping characteristics and to the variability of the variance of the process since

$$
\Gamma_{\eta}(0)=4 T_{\nu}=Q_{e} T_{01}
$$

where $T_{\nu}=$ a temporal spectral peakedness parameter introduced by Medina et a1. (1985); and $Q_{e}=$ a dimensionless spectral peakedness parameter proposed by Medina and Hudspeth (1987). This peakedness parameter is similar to the Goda peakedness parameter, $Q_{p}$, and is also related to parameters introduced by Tucker (1963) and Blackman and Tukey (1959). The $Q_{e}$ parameter is defined as

$$
Q_{e}=\frac{2 m_{1}}{m_{0}^{3}} \int_{0}^{\infty} S_{\eta}^{2}(f) d f
$$

Finally, the envelope of the autocorrelation function has also been used to analyze wave groups.

\section{Envelope of the Autocorrelation Function (Rye, 1982)}

Figure 3 illustrates the spectrum autocorrelation function and corresponding envelope for a rectangular and for a Goda-JONSWAP $\operatorname{spectra}(\gamma=3.3)$. 
Because the rectangular-shaped spectrum demonstrates a local maximum in the envelope of the autocorrelation function at $r=1.5 / \mathrm{B}$ where $\mathrm{B}=$ rectangular spectral bandwidth, Rye (1982) attempted to analyze wave groups using the envelope of the autocorrelation function.

\section{[INSERT FIG. 3 HERE]}

Note that in Fig. 3b the envelope of the autocorrelation function for the Goda-JONSWAP spectrum decreases monotonically. Because of this monotonic behavior and because of the statistical variability in real ocean wave records, Rye was not successful in using the local maxima of envelope of the autocorrelation function to characterize wave groups.

However, it will be shown in the following that the envelope of the autocorrelation function is related to the spectrum of the wave height function. Therefore, the envelope of the autocorrelation function may be used to characterize wave groups.

\section{WAVE HEIGHT FUNCTION ANALYSIS}

Assuming that the sea surface elevation at a point is an ergodic Gaussian stochastic process having a variance spectrum, $S_{\eta}(f)$, a realization may be approximated by

$$
\eta(t)=\sum_{m=1}^{M} R_{m} \cos \left(2 \pi f_{m} t+\theta_{m}\right)
$$

where $M=$ the total number of wave components in the realization; $R_{m}, f_{m}$, and $\theta_{\mathrm{m}}=$ the amplitude, the frequency, and a random phase angle, respectively, of the $\mathrm{m}^{\text {th }}$. wave component. The random phase angle is uniformly distributed in the interval $U[0,2 \pi]$. The Hilbert transform, $\hat{\eta}(t)$, of $\eta(t)$ (Bendat and Piersol, 1986), is given by 


$$
\hat{\eta}(t)=\sum_{m=1}^{M} R_{m} \sin \left(2 \pi f_{m} t+\theta_{m}\right)
$$

and the analytical function by

$$
A F(t)=\eta(t)+j \hat{\eta}(t)=A(t) \exp (j[\theta(t)+\phi])
$$

where $j=\sqrt{-1} ; \quad A(t)=$ the envelope function; and $[\theta(t)+\phi]=$ the instantaneous phase angle defined by

$$
A(t)=\left[\eta^{2}(t)+\hat{\eta}^{2}(t)\right]^{1 / 2} ; \theta(t)+\phi=\operatorname{ARCTAN}[\hat{\eta}(t) / \eta(t)]
$$

In the complex plane, Hudspeth and Medina (1988) identified three interpretations of $A F(t):$ 1) a vertical displacement of a point floating in the sea surface, 2) a horizontal displacement, and 3) an orbital movement. An instantaneous wave height, $H(t)=2 A(t)$, and a local frequency, $\Omega(t)=$ $d \theta(t) / d t$, were defined. The statistical properties of these two functions were evaluated and related to characteristics of wave groups.

From the definition of $H^{2}(t)$ and its spectrum, $S_{\mathrm{H}_{2}}(f)$, the inverse of the mean frequency of $S_{\mathrm{H} 2}(f)$ can be interpreted as the average mean period, ${ }_{2} \bar{T}_{\mathrm{H}_{\text {rms }}}$, of $H^{2}(t)$ which can be normalized by the mean wave period, $T_{01}$, to obtain the average length of a total run of waves at a threshold level $h=H_{\text {rms }}$ (vide Eq. (37)).

[INSERT FIG. 4 HERE]

Figure 4 illustrates different envelope spectral density functions (unit variance) normalized by $6 \mathrm{~T}_{\nu}$. The normalized mean period, ${ }_{2} \bar{\tau}_{\mathrm{H}_{\mathrm{rms}}} / 6 \mathrm{~T}_{\nu}$, of $\mathrm{H}^{2}(\mathrm{t})$ is equal to unity in Fig. 4 for the rectangular spectrum. Therefore, for the rectangular spectrum the average length of a total run of waves at $h=H_{r m s}$ may be approximated by

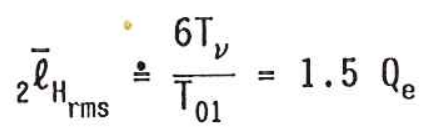


On the other hand, the normalized envelope spectral density functions for the Goda-JONSWAP spectra in Fig. 4 have normalized mean periods, ${ }_{2} \bar{T}_{\mathrm{r}_{\mathrm{rms}}} / 6 \mathrm{~T}_{\nu}$, which are smaller than unity. As a consequence, the expected length of a total run of waves at $h=H_{\text {rms }}$ are lower than for the rectangular spectrum. Therefore, the expected length of a total run of waves for real wave spectra will be proportional to the estimate for a rectangular spectrum according to

$$
{ }_{2} \bar{\ell}_{H_{\text {rms }}} \doteq 1.5 Q_{\mathrm{e}} \mathrm{C}_{\mathrm{e}}
$$

where $C_{e}=$ a constant of proportionality that is a function of the spectral shape and the Nyquist frequency $f_{N}=1 / 2 \Delta T$ where $\Delta T=$ the sampling time interval of the squared-wave height function. In order to compare estimates of run lengths given by Eq. (47) with observations of run lengths computed from discrete waves, the value of $\Delta T$ should be approximately equal to $T_{01}$. because discrete wave heights should be comparable with values of $H(t)$ discretized at this time interval.

Figure 5 illustrates the effect of spectral shape $(\gamma=1.0,3.3$, and 10.0) and of sampling time intervals $\left(\Delta T=T_{\nu}\right.$ and $\left.T_{\nu} / 2\right)$ on the constant of proportionalities, $C_{e}$, for the Goda-JONSWAP spectrum.

[INSERT FIG. 5 HERE]

The similarity between the spectral peakedness parameters, $Q_{p}$ and $Q_{e}$, defined by Eqs. (1) and (41) appears to be useful for comparing methods for data analyses. For the Goda-JONSWAP spectra, the ratio $Q_{p} / Q_{e} \doteq 0.87$ with a slight tendency to increase with increasing values of $\gamma$.

Following the ideas presented in Eqs. (38) and (39) to characterize wave groups by an envelope spectral density function (unit variance), $\Gamma_{\eta}(f)$, it is reasonable to consider the Fourier transform of $\Gamma_{\eta}(f)$ as a potentially useful 
function. The Fourier transform of $\Gamma_{\eta}(f)$ is the square of the envelope of the autocorrelation function of the stochastic process (Bendat and Piersol, 1986).

\section{[INSERT FIG. 6 HERE]}

Figure 6 illustrates the Wiener-Khinchine relations for a) waves and b) envelopes. Figure 6 a shows the unit variance spectrum, $S_{\eta}(f) / m_{0}$, the corresponding autocorrelation function, $\mathrm{R}_{\eta}(\tau)$, and its envelope, $A_{R_{\eta}}(\tau)$; and the Hilbert transform of the autocorrelation function, $\hat{\mathrm{R}}_{\eta}(\tau)$. Similarly, Fig. $6 \mathrm{~b}$ shows the envelope spectral density function (unit variance), $\Gamma_{\eta}(f)$; its Fourier transform, $A_{R_{\eta}}^{2}(r)=R_{H_{2}}(\tau)$; as well as the square of the autocorrelation function, $\mathrm{R}_{\eta}^{2}(\tau)$, and the square of its Hilbert transform, $\hat{\mathrm{R}}_{\eta}^{2}(\tau)$. Note that the autocorrelation function of the squared envelope, $\hat{R}_{\mathrm{H} 2}(\tau)$, is the square of the envelope of the autocorrelation function, $A_{R_{\eta}}^{2}(\tau)$.

Even though Rye (1982) was unable to find any useful properties (i.e., no local maxima) in the envelope of the autocorrelation function, $A_{R_{\eta}}(\tau)$, the following analysis appears to support the hypothesis that the square of the envelope of the autocorrelation function, $A_{R_{\eta}}^{2}(\tau)$, should contain the basic properties of the characteristics of the wave groups in a stochastic process. Note that by Eqs. (38), $\Gamma_{\eta}(f)$ is also an approximation for the spectral density function (unit variance) of the wave height function, $H(t)$. Its Fourier transform, $A_{R_{\eta}}^{2}(\tau)$, should be an approximation for the autocorrelation function of the envelope, $R_{A}(\tau)$. Therefore, according to Eqs. (13), (25), and (27), we find

$$
\begin{aligned}
& A_{\mathrm{R}_{\eta}}^{2}(\tau) \doteq \mathrm{R}_{\mathrm{A}}(\tau)=\mathrm{R}_{\mathrm{H}}(\tau) \doteq \mathrm{R}_{\mathrm{H} 2}(\tau) \\
& \mathrm{A}_{\mathrm{R}_{\eta}}^{2}\left(\mathrm{~T}_{01}\right) \doteq \kappa^{2}=(2 \rho)^{2}
\end{aligned}
$$

where $\kappa=$ the parameter defined in Eq. (19) used by Longuet-Higgins (1984) in Eq. (25); and $2 \rho=$ the parameter used by Kimura (1980) in Eq. (3). The 
parameter, $\kappa$, used by Battjes and Vledder (1984) in a formula similar to Eq. (25) was defined using the envelope of the autocorrelation function; $A_{R_{\eta}}(\tau)$, and $T_{02}$ instead of $T_{01}$ as the mean period.

\section{[INSERT FIG. 7 HERE]}

Figure 7 illustrates the Wiener-Khinchine relationships for the envelope spectra for different values of $\gamma$ for the Goda-JONSWAP spectrum. The envelope spectral density functions (unit variance) have been normalized by $T_{01}$. In principle, the square of the envelope of the autocorrelation function in Fig. $7 b, R_{H 2}\left(\tau / T_{01}\right) \doteq A_{R_{\eta}}^{2}\left(\tau / T_{01}\right)$, could be used in Eq. (49) to estimate wave groupiness parameters.

\section{STATISTICS OF RUN LENGTHS}

Two simple approximations have been used to define the probability density function (p.d.f.) for the length of run of high waves and the total run of waves: viz. 1) the exponential and 2) the Kimura approximations. The first approximation is based on the independence between crossings of the wave height function at a given threshold level. The second is based on a Markov chain hypothesis for the discrete wave heights as given by Eqs. (6) to (9).

\section{Exponential Approximation}

The exponential approximation may be derived from a Poisson distribution (cf. Longuet-Higgins, 1984). Assuming that successive upcrossings of $H(t)$ at a given threshold level are uncorrelated (which is reasonable for narrowbanded processes and for large ${ }_{2} \bar{\ell}_{h}$ ), then

$$
1-P\left({ }_{2} \ell_{h}\right)=\lim _{m \rightarrow \infty}\left[1-\frac{1}{m}\right]^{\left[{ }_{2} \ell_{h} /\left({ }_{2} \bar{l}_{h} / m\right)\right]}
$$


where $P\left({ }_{2} \ell_{h}\right)=$ the probability that the length of a total run of waves is less than ${ }_{2} \ell_{h} ;{ }_{2} \bar{\ell}_{h}=E\left[{ }_{2} \ell_{h}\right]=$ the mean 1ength of a total run of waves; and $\left({ }_{2} \bar{l}_{h} / \mathrm{m}\right)=$ a small subinterval of ${ }_{2} \bar{l}_{h}$ which has a very low probability $(=1 / \mathrm{m})$ of detecting an upcrossing. In the limit as $m \rightarrow \infty$,

$$
1-P\left({ }_{2} \ell_{h}\right)=\exp \left[-\left({ }_{2} \ell_{h} /{ }_{2} \bar{\ell}_{h}\right)\right]
$$

and, therefore

$$
p\left({ }_{2} \ell_{h}\right)=\left(1 /{ }_{2} \bar{\ell}_{h}\right) \exp \left[-\left({ }_{2} \ell_{h} /{ }_{2} \bar{\ell}_{h}\right)\right]
$$

in which $p\left({ }_{2} l_{h}\right)=$ the probability density function of the length of a total run of waves.

With similar assumptions, analogous approximations for $P\left({ }_{1} \ell_{h}\right)$ and $p\left({ }_{1} \ell_{h}\right)$ yield;

$$
\begin{aligned}
& P\left({ }_{1} \ell_{h}\right)=1-\exp \left[-\left({ }_{1} \ell_{h} / \bar{l}_{h}\right)\right] \\
& P\left({ }_{1} \ell_{h}\right)=\left(1 /{ }_{1} \bar{\ell}_{h}\right) \exp \left[-\left({ }_{1} \ell_{h} / \bar{\ell}_{h}\right)\right]
\end{aligned}
$$

If the wave height function is Rayleigh distributed, then a relationship between the average run lengths is given by

$$
\left[{ }_{2} \bar{\ell}_{h} / \bar{\ell}_{1}\right]=\frac{1}{1-P(h)}=\exp \left[h^{2} / 8 m_{0}\right]
$$

where ${ }_{2} \bar{\ell}_{h}$ and ${ }_{1} \bar{\ell}_{h}=$ the average values for the length of a total run of waves and a run of high waves for a threshold level $h$, respectively; and $P(h)=$ the cumulative distribution function (c.d.f.) of the wave heights. Note that Eq. (55) is equivalent to Eq. (34).

\section{Three-Axes Representation}

Both the exponential [Eqs. (51-55)] and the Markov Chain approximations [Eqs. (6-9)] yield p.d.f.'s for ${ }_{1} l_{h}$ and ${ }_{2} l_{h}$ that require knowing only the average lengths of runs, ${ }_{1} \bar{\ell}_{h}$ and ${ }_{2} \bar{\ell}_{h}$. Therefore, estimates for the pairs of 


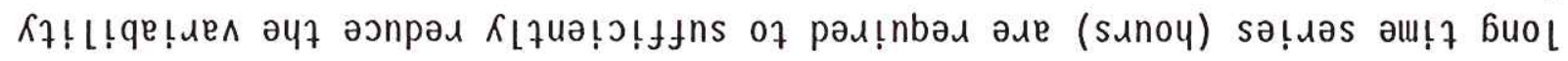

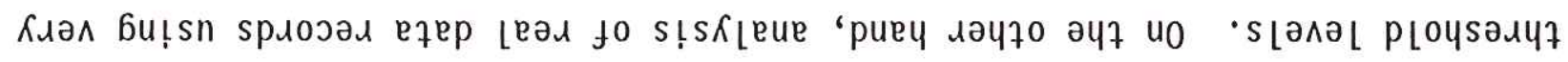

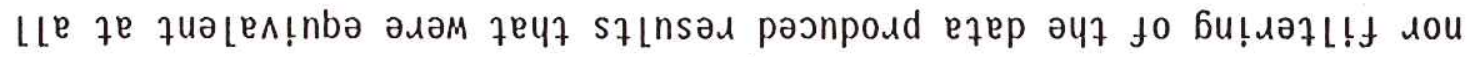

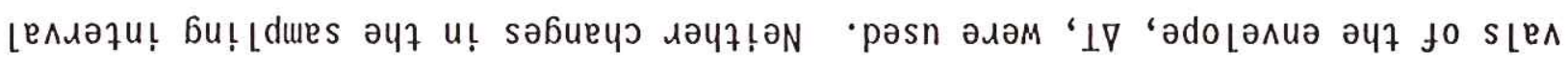

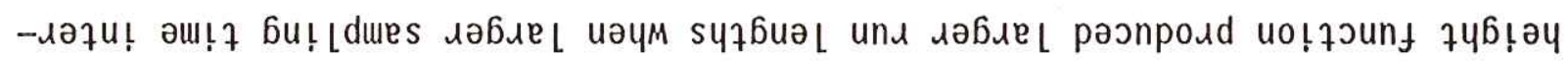

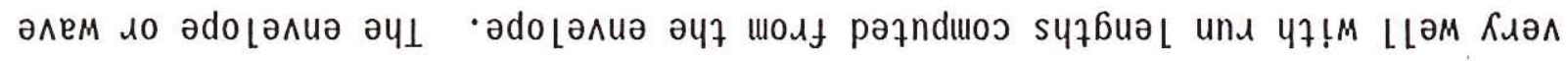

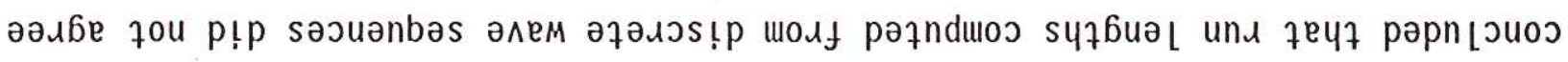

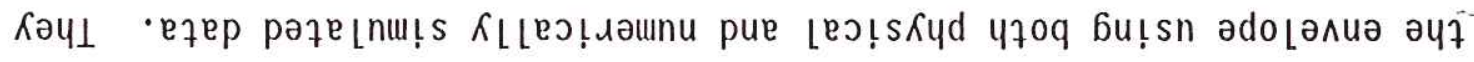

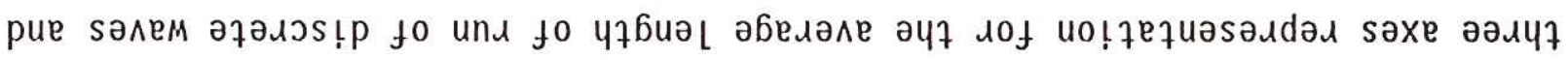

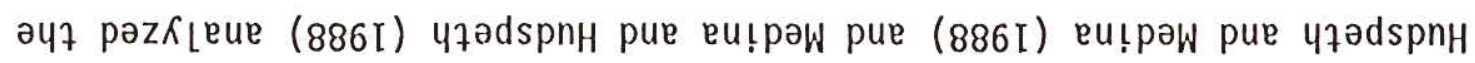

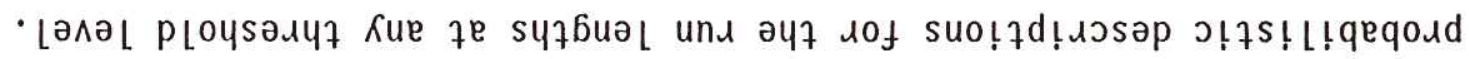

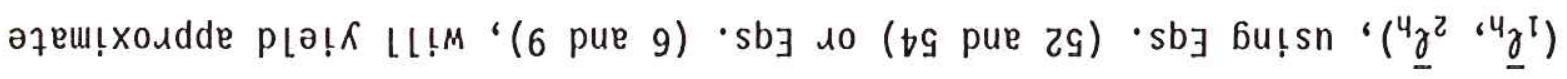

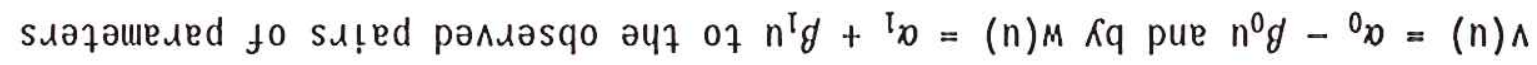

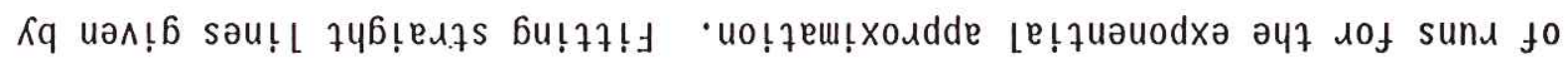

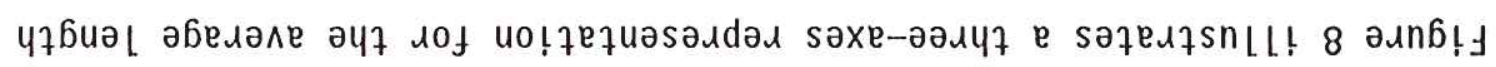

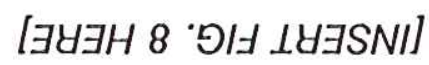

- sәлем to sund buol of puodsaldos

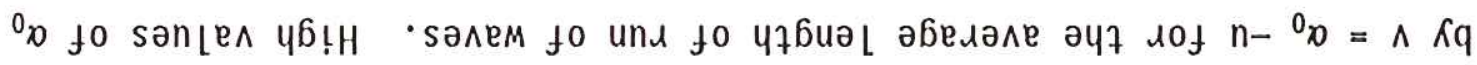

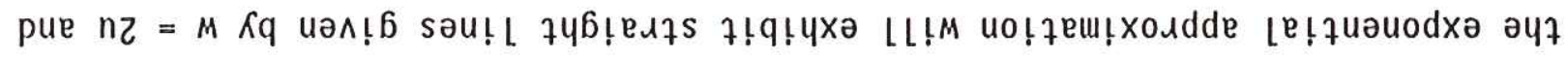
$\left(\rho^{\prime} q^{\prime} \mathrm{egq}\right)$

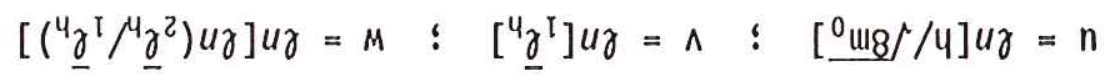

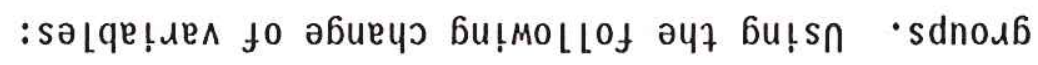

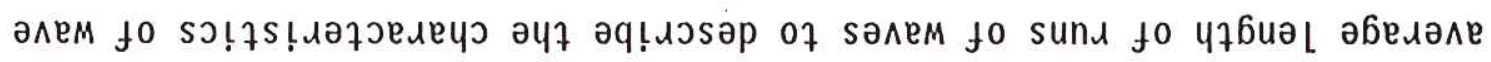

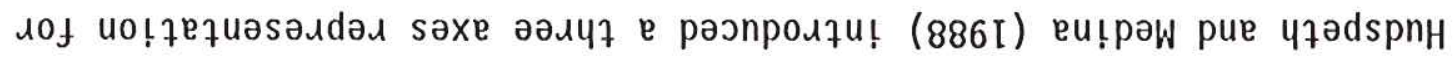
-syzbual und fo sว!zs!zezs әч7 u!̣еqqo

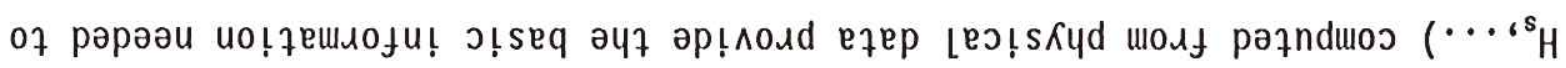

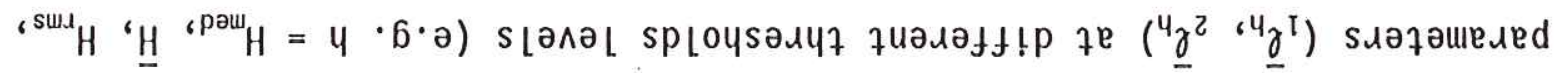


in the estimates of the parameters ${ }_{1} \bar{l}_{h},{ }_{2} \bar{l}_{h}$ (envelope), or ${ }_{1} \bar{L}_{h},{ }_{2} \bar{L}_{h}$ (discrete waves) in order to realize reliable statistics of run lengths.

\section{CORRELATION BETWEEN SUCCESSIVE WAVE HEIGHTS}

Analyzing wave groups in long-traveled swe11 waves, Goda (1983) pointed out that the correlation coefficient between consecutive wave heights, $r_{H H}(1)$, is a better parameter to define the length of runs of wave heights than is the spectral peakedness parameter, $a_{p}$. On the other hand, he noted that $r_{H H}(1)$ is probably correlated with $Q_{p}$ and could, therefore, be considered as an internal parameter to describe the phenomenon of wave grouping. We demonstrate this correlation later in Fig. 11.

[INSERT FIG. 9 HERE]

If $\Gamma_{\eta}(f)$ is approximately the spectral density function of the wave height function, then its Wiener-Khinchine transform pair illustrated in Fig. $7 \mathrm{~b}$ should be approximately the autocorrelation function of the wave height function. Therefore, a correlation between successive wave heights could be estimated from the Wiener-Khinchine pair. Figure 9 represents estimations of $r_{H H}(m)$ from Goda-JONSWAP spectra using Eq. (48) and values from the WienerKhinchine pairs illustrated in Fig. 7.

[INSERT FIG. 10 HERE]

It appears reasonable that values of $r_{H H}(1)$ computed from discrete waves should be approximately equivalent to values of $r_{H H}(1)$ computed from wave height envelopes discretized at $\Delta T=T_{01}$. Figure 10 compares three sets of observed values for $r_{H H}(m)$ computed from long-traveled swell waves by Goda (1983) with theoretical estimates computed from Goda-JONSWAP spectra using two 
cut-off frequencies. These two theoretical estimates for $R_{H}(\tau)$ were obtained from two sampling intervals; viz. $\Delta T=T_{01} / 16 \& \Delta T=T_{01}$.

[INSERT FIG. 11 HERE]

Five of the parameters listed in Table 2 to characterize wave groups may be related to each other in a simple manner. Figure 11 illustrates this simple theoretical relationship using Eqs. $(13,17$, and 48$)$ and the WienerKhinchine pairs shown in Fig. 7. the theoretical relationships were computed from Goda-JONSWAP spectra for 6 values of the peak enhancement factor, $\gamma$, using two different values for the cut-off frequency for the envelope spectra given by $f_{N}^{-1}=2 \Delta T$ for $\Delta T=T_{01}$ and $T_{01} / 16$. The first quadrant $\left[r_{H H}(1) v s . Q_{p}\right]$ compares the observations of long-traveled swe11 waves and the numerical simulations of Kimura that were reported by Goda (1983) with theoretical estimates computed using the two cut-off frequencies $\left(\Delta T=T_{01}\right.$ and $\left.T_{01} / 16\right)$. The second quadrant $\left[Q_{p}\right.$ vs. $\left.Q_{e}\right]$ demonstrates the close similarity $\left(Q_{p} / Q_{e} \cong\right.$ 0.87 ) between the spectral peakedness parameters computed by Eqs. (1 and 41). The third quadrant $\left[Q_{p}\right.$ vs. $\left.\kappa^{2}\left[=(2 \rho)^{2}\right]\right]$ illustrates theoretically using Eq. (49) the relationship between these two parameters as a function of the two cut-off frequencies for the envelope spectra. Finally, the fourth quadrant $\left[\kappa^{2}\left[=(2 \rho)^{2}\right]\right.$, vs, $\left.r_{H H}(1)\right]$ presents the relationship between these two parameters defined in Eq. (27).

of course, estimates of the 5 parameters that are related theoretically in Fig. 11 will be less correlated when computed from real ocean wave data due to the statistical variability of the functions used to estimate them.

\section{SUMMARY AND CONCLUSIONS}

The parameters and functions derived from the most commonly used methodologies to characterize wave groups in linear waves have been reviewed and 
interrelated in a unified manner. The run length methodology and the exponential and Markov chain approximations have been interrelated in a three-axes representation of run lengths. A second interrelationship was made for the envelope theory; for the wave height function theory; for the $\eta^{2}(t)$ filter theories; and for the correlation function theories. Finally, the correlation coefficient between consecutive wave heights $\left(r_{H H}(1)\right)$ was shown to be highly correlated with the spectral peakedness parameters $\left(Q_{p}\right.$ and $\left.Q_{e}\right)$.

The wave height function method was reviewed in some detail in order to demonstrate the relationship between the run lengths theory and the envelope theories. A three-axes representation demonstrated that run lengths computed from a discrete wave height method did not correlate with run lengths computed from a continuous envelope.

Four parameters $\left(Q_{p}, Q_{e}, \kappa^{2}=4 \rho^{2}\right)$ used to characterize wave groups were compared with the correlation coefficient $\left(r_{H H}(1)\right)$ for order to demonstrate that each parameter was useful. However, because of their interrelationship, only one of the parameters or the correlation coefficient is independent.

Finally, run lengths and any of the wave group parameters must be estimated from a linear random process; and are, therefore, subject to a statistical variability. Very long data records are required in order to reduce to an acceptable level the statistical variability of estimates of these parameters and functions. The theoretical estimates provided in this review must be interpreted as first-order trends to the estimates computed from real data.

\section{ACKNOWLEDGEMENTS}

We gratefully acknowledge the financial support provided by the U.S.Spain Joint Committee for Scientific and Technological Cooperation, under Grant CCA8510095. Robert T. Hudspeth was supported by the Office of Naval 
Research under the University Research Initiative (URI) Contract No. N0001486-K-0687. Numerical results were provided by Claudio Fassardi.

\section{REFERENCES}

Abramowitz, M. and Stegun, I.A. (1968): Handbook of Mathematical Functions, Dover Publications, Inc., (p. 376, 590).

Agnon, Y. and Mei, C.C. (1988): "Excitation of Long Internal Waves by Groups of Short Surface Waves Incident on a Barrier, "Journal of Fluid Mechanics, Vol. 192, pp. 17-31.

Arhan, M. and Ezraty, R. (1978): "Statistical Relations Between Successive Wave Heights," Oceanologica Acta, Vol. 1, No. 2, pp. 151-158.

Blackman, R.B. and Tukey, J.W. (1959): The Measurement of Power Spectra, Dover Publications, 1959, pp. 19-21.

Battjes, J.A., and Vledder, V. (1984): "Verification of Kimura's Theory for Wave Group Statistics, "Proceedings, 19th ICCE, 1984, Houston, TX, pp. 642-648 Battjes, J.A. (1988): "Surf-Zone Dynamics," Annual Review Fluid Mechanics, No. 20, pp. 257-293.

Bendat, J.S. and Piersol, A.G. (1986): Random Data. Analysis and Measurement Procedures, (2nd Ed.), John Wiley, pp. 484-516.

Bowers, E.C. (1977): "Harbour Resonance Due to Set-Down Beneath Wave Groups," Journal of Fluid Mechanics, Vol. 29, Part 1, pp. 71-92.

Bowers, E.C. (1988): "Wave Grouping and Harbour Design," Proceedings of the Institution of Civil Engineers, Part 2, Vo1. 85, June 1988, pp. 227-258.

Bruun, P. (ed.), (1985): Design and Construction of Mounds for Breakwaters and Coastal Protection, Elsevier Science Pub1., Amsterdam, The Netherlands.

Burchart, H.F. (1979): "The Effect of Wave Grouping on On-Shore Structures," Coastal Engineering, No. 2, pp. 189-199.

Elgar, S., Guza, R.T., and Seymour, R.J. (1984): "Groups of Waves in Shallow Water," Journal of Geophysical Research, Vol. 89, No. C3, pp. 3623-3634.

Elgar, S., Guza, R.T., and Seymour, R.J. (1985): "Wave Group Statistics from Numerical' Simulations of a Random Sea," Applied Ocean Research, Vol. 7, No. 2, pp. 93-96.

Ewing, J.A. (1973): "Mean Length of Runs of High Waves, "Journal of Geophysical Research, Vol. 78, No. 12, pp. 1933-1936.

Funke, E.R. and Mansard, E.P.D. (1979): "On the Synthesis of Realistic Sea States in a Laboratory Flume," HLR Report LTR-HY 66, National Research Council of Canada, Ottawa. 
Goda, Y. (1970): "Numerical Experiments on Wave Statistics with Spectral Simulation," Report of the Port and Harbour Research Institute, Vo1. 9, No. 3, 1970 .

Goda, Y. (1976): "On Wave Groups," Proceedings, Conference on Behaviour of offshore Structures (BOSS), Trondheim, Vol. I, pp. 115-128.

Goda, Y. (1983): "Analysis of Wave Grouping and Spectra of Long-Traveled Swe11," Report of the Port and Harbour Research Institute, Vol. 22, No. 1, 1983.

Hsu, F.H. and Blenkarn, K.A. (1970): "Analysis of Peak Mooring Forces Caused by Slow Vessel Drift Oscillations in Random Seas," Proceedings, OTC Conference, Houston, TX, 1970, Paper No. 1159.

Hudspeth, R.T., and Medina, J.R. (1988): "Wave Groups Analyses by the Hilbert Transform," 21st ICCE, 1988, Torremolinos, Spain (in press).

Johnson, R.R., Mansard, E.P.D., and Ploeg, J. (1978): "Effects of Wave Grouping on Breakwater Stability," Proceedings, 16th ICCE, 1978, Hamburg, Germany, pp. 2228-2243.

Kimura, A., (1980): "Statistical Properties of Random Wave Groups," Proceedings, 17th ICCE, 1980, Sydney, Australia, pp. 2955-2973.

Longuet-Higgins, M.S. (1957): "The Statistical Analysis of a Random, Moving Surface," Phi1. Trans. Roy. Soc. London, Ser. A (1966), Vol. 249, pp. 321-387.

Longuet-Higgins, M.S. (1984): "Statistical Properties of Wave Groups in a Random Sea State," Phil. Trans. Royal Soc. London, A312, pp. 219-250.

Mase, M., and Iwagaki, Y. (1986): "Wave Group Analysis from Statistical Viewpoint," Proceedings, Ocean Structural Dynamics Symposium '86, Corvallis, OR, pp. 145-157.

Medina, J.R., Aguilar, J., and Diez, J.J. (1985): "Distortions Associated with Random Sea Simulators," Journal of Waterway, Port, Coastal and Ocean Engineering, ASCE, Vol. 111, No. 4, pp. 603-628.

Medina, J.R. and Hudspeth, R.T. (1987): "Sea States Defined by Wave Height and Period Functions," Proceedings, IAHR Seminar on Wave Analys is and Generation in Laboratory Basins, 22nd IAHR Congress, Lausanne, Switzerland, pp. 249259.

Medina, J.R. and Hudspeth, R.T. (1988): "Analysis of Wave Groups in Random Fields," Proceedings, Ocean Structural Dynamics Symposium '88, Corvallis, OR, pp. 104-118.

Nolte, K.G. and Hsu, F.H. (1972): "Statistics of Ocean Wave Groups," Proceedings, OTC Conference, Houston, TX, OTC Paper No. 1688.

Rice, S.0. (1954): "Mathematical Analysis of Random Noise," Bell System Technical Journal, Vo1. 23, 1944, and Vo1. 24, 1945. (Reprinted in Selected Papers on Noise and Stochastic Processes, N. Wax, Ed., Dover Publications, Inc., New York, NY, 1954, pp. 123-244.) 
Rye, H. (1974): "Wave Group Formation Among Storm Waves," Proceedings, 14th ICCE, 1974, Copenhagen, Denmark, pp. 764-783.

Rye, H. (1982): Ocean Wave Groups, Dept. Marine Technology, Norwegian Institute of Technology, Report UR-82-18.

Sand, S.E. (1982): "Long Waves in Directional Seas," Coastal Engineering, Vo1. 6, pp. 195-208.

Sand, S.E. (1982): "Wave Grouping Described by Bounded Long Waves, "Ocean Engineering, Vol. 9, №. 6, pp. 567-580.

Sand, S.E. (1982): "Long Wave Problems in Laboratory Mode1s," Journal of Waterway, Port, Coastal and Ocean Division, ASCE, Vol. 108, No. WW4, pp. 492503.

Thompson, E.F. and Seelig, W.N. (1984): "High Wave Grouping in Shallow Water," Journal of Waterway, Port, Coastal and Ocean Engineering, ASCE, Vol. 110, No. 2, pp. 139-157.

Tucker, M.J. (1950): "Surf Beats: Sea Waves of 1 to 5 min. Period," Proceedings of the Royal Society, A, Vo1. 202, pp. 565-573.

Tucker, M.J. (1963): "Analysis of Records of Sea Waves, "Proceedings, Institute of Civil Engineers, Vol. 26, No. 10, pp. 305-316.

Vanmarcke, E. (1972): "First Passage of Nonstationary Random Processes," Journal of the Engineering Mechanics Division, ASCE, Vol 98, No. EM2, pp. 401-414.

Vanmarcke, E. (1983): Random Fields, MIT Press, Cambridge, MA, 1983, pp. 139-183. 


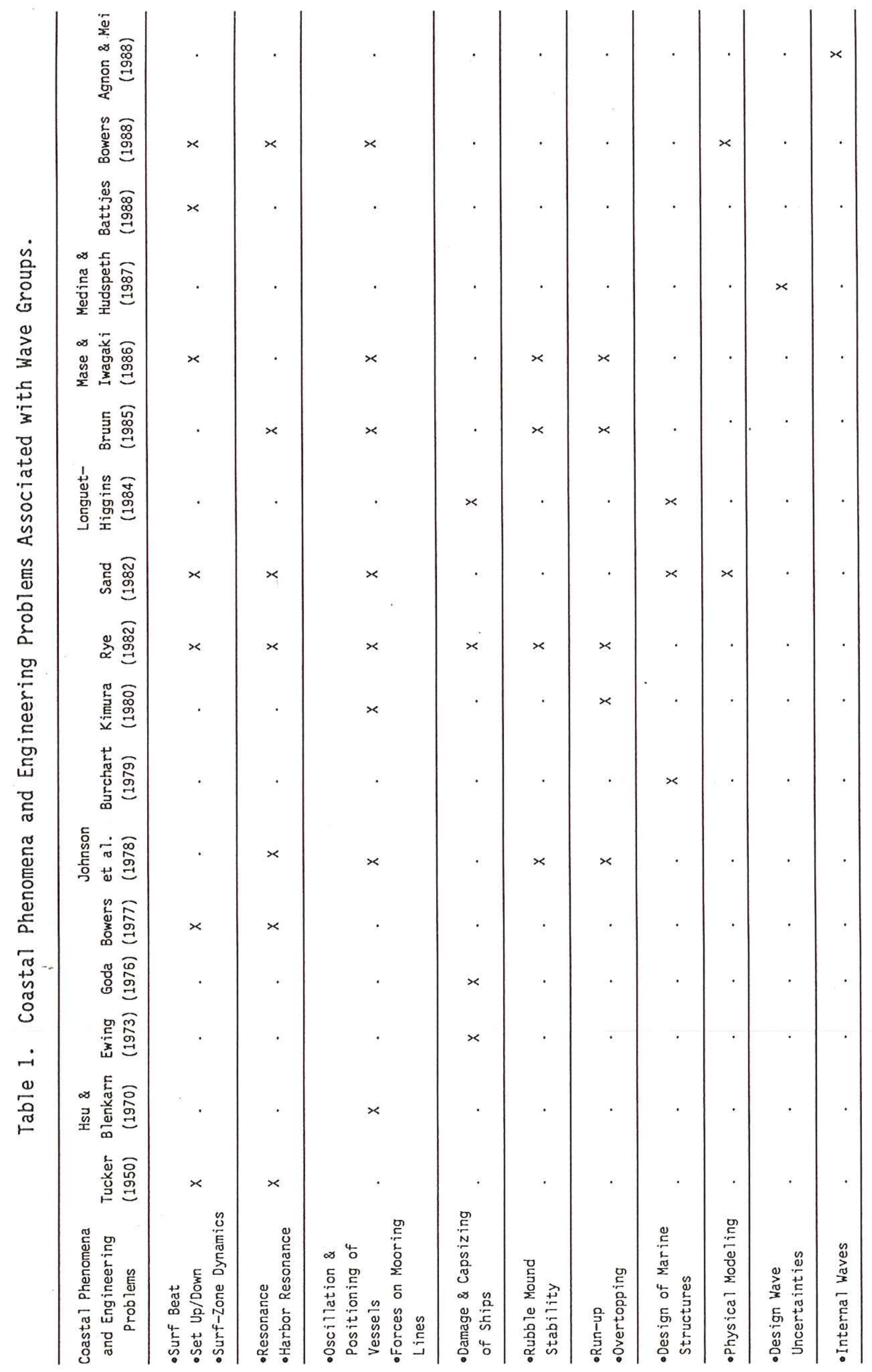




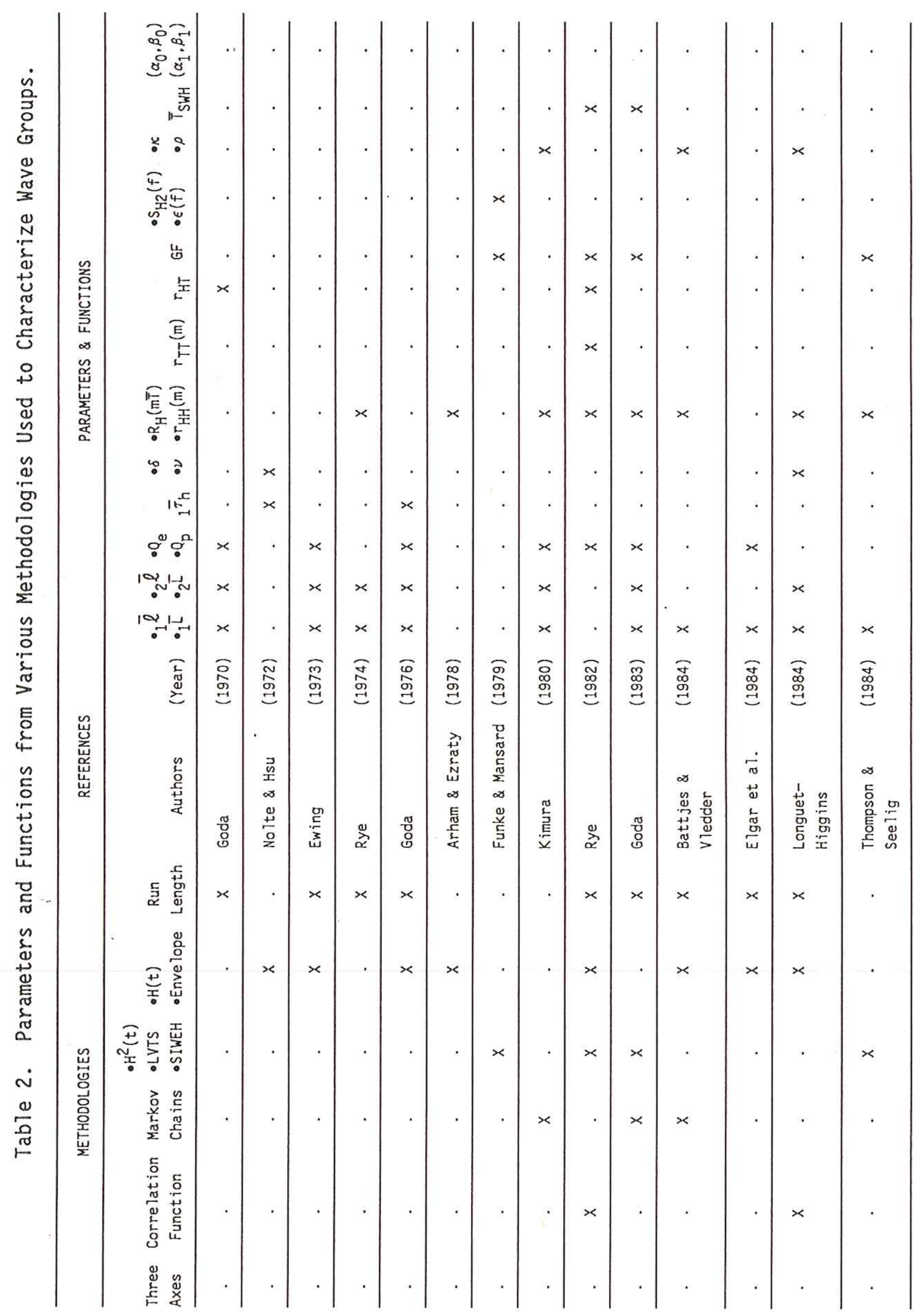




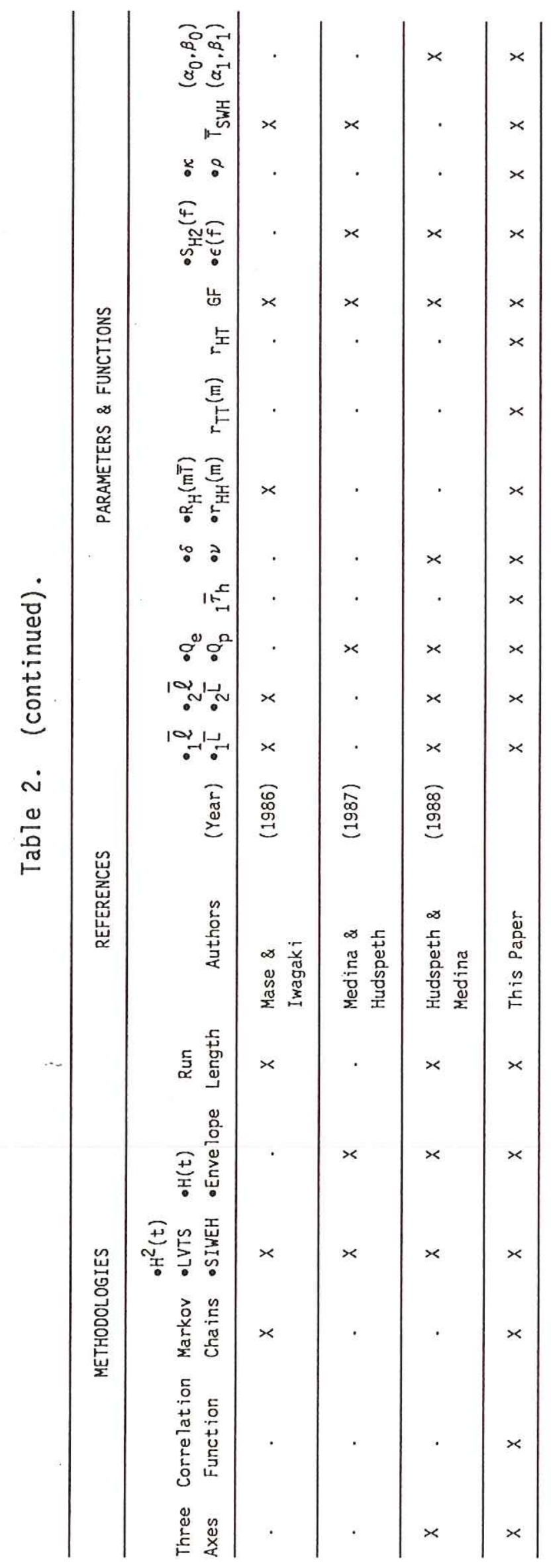



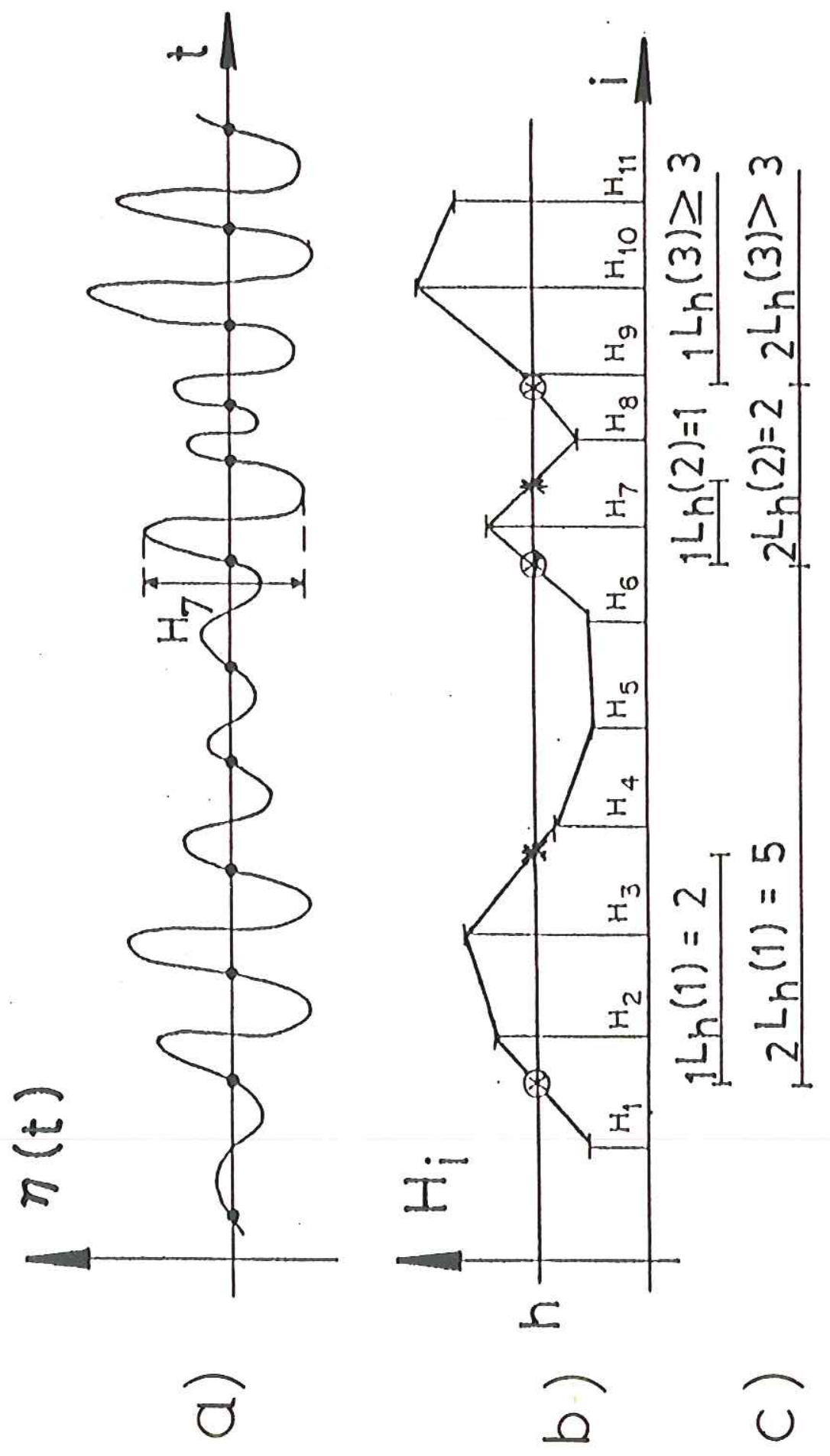

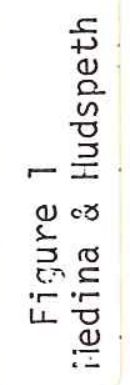




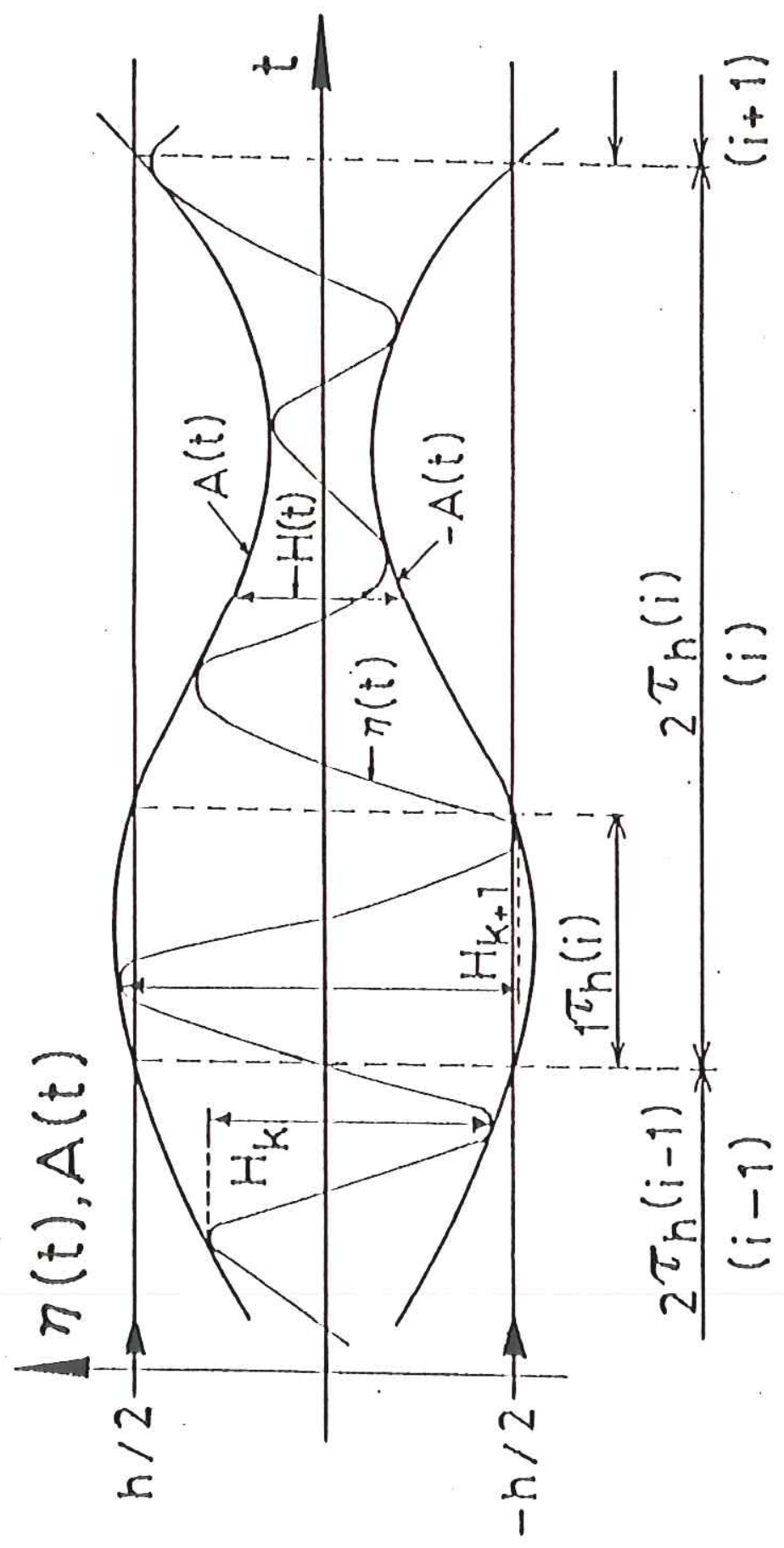

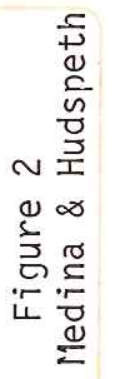



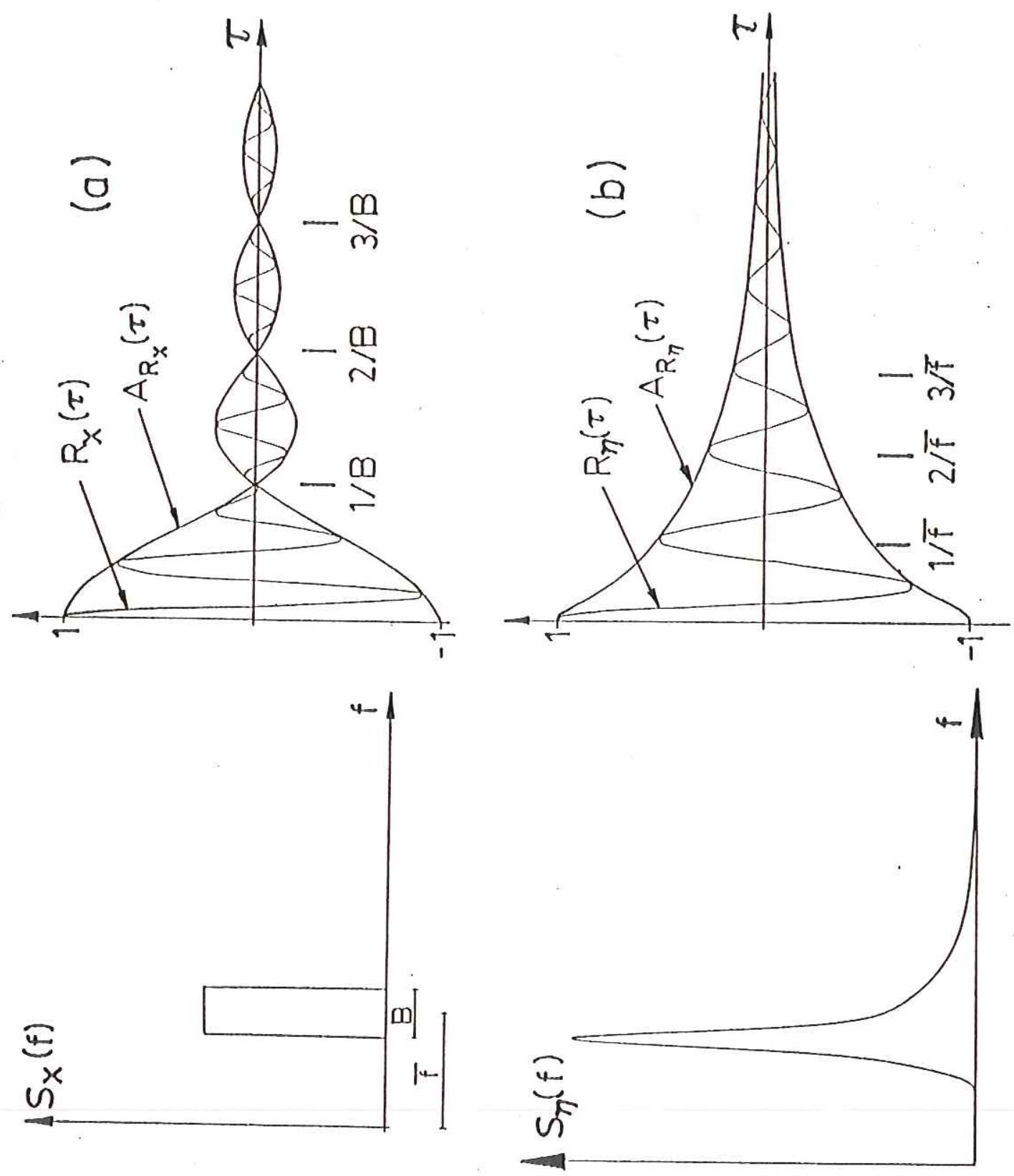

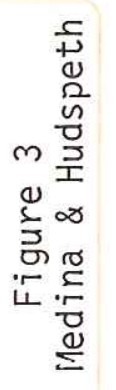




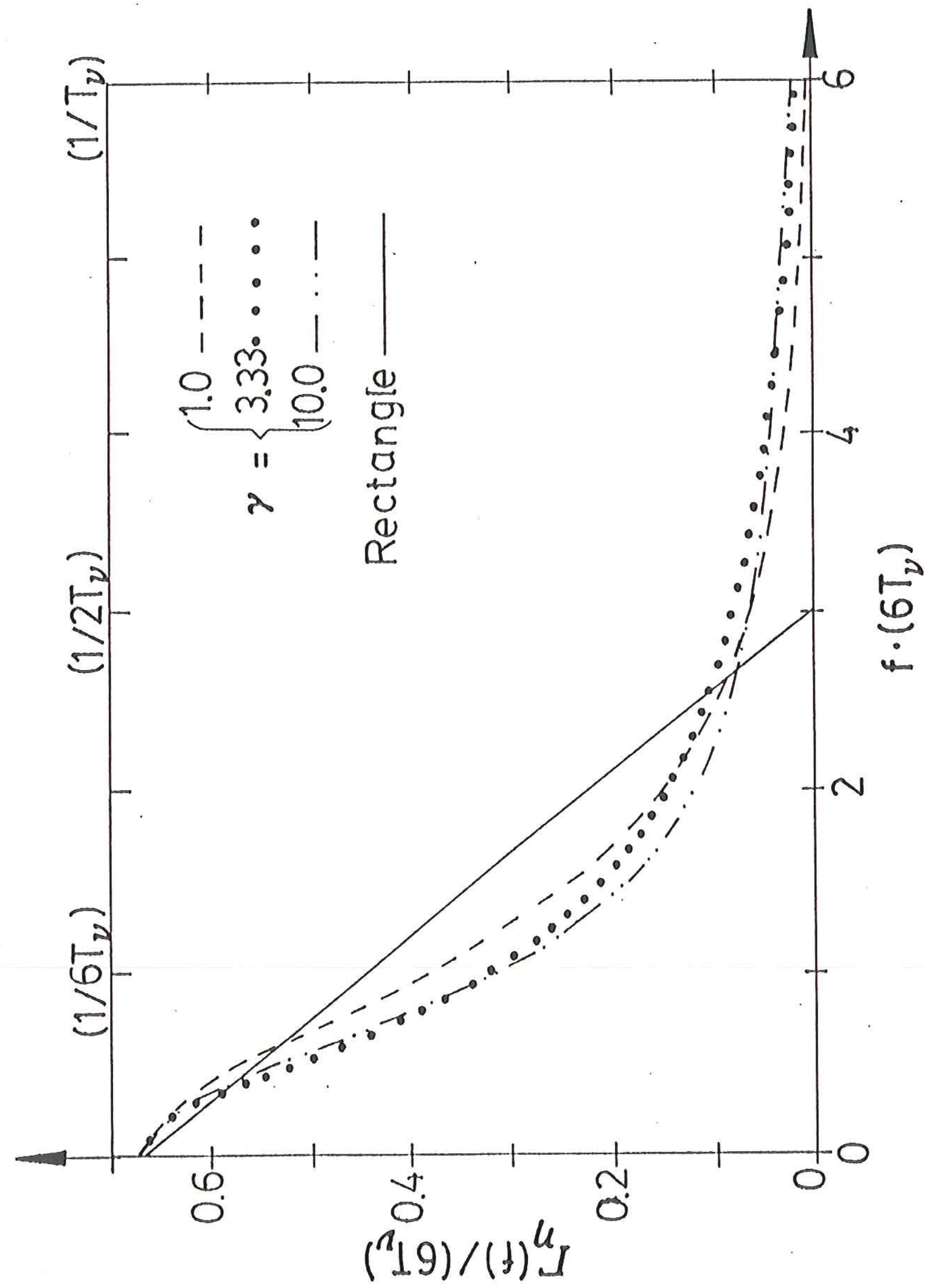




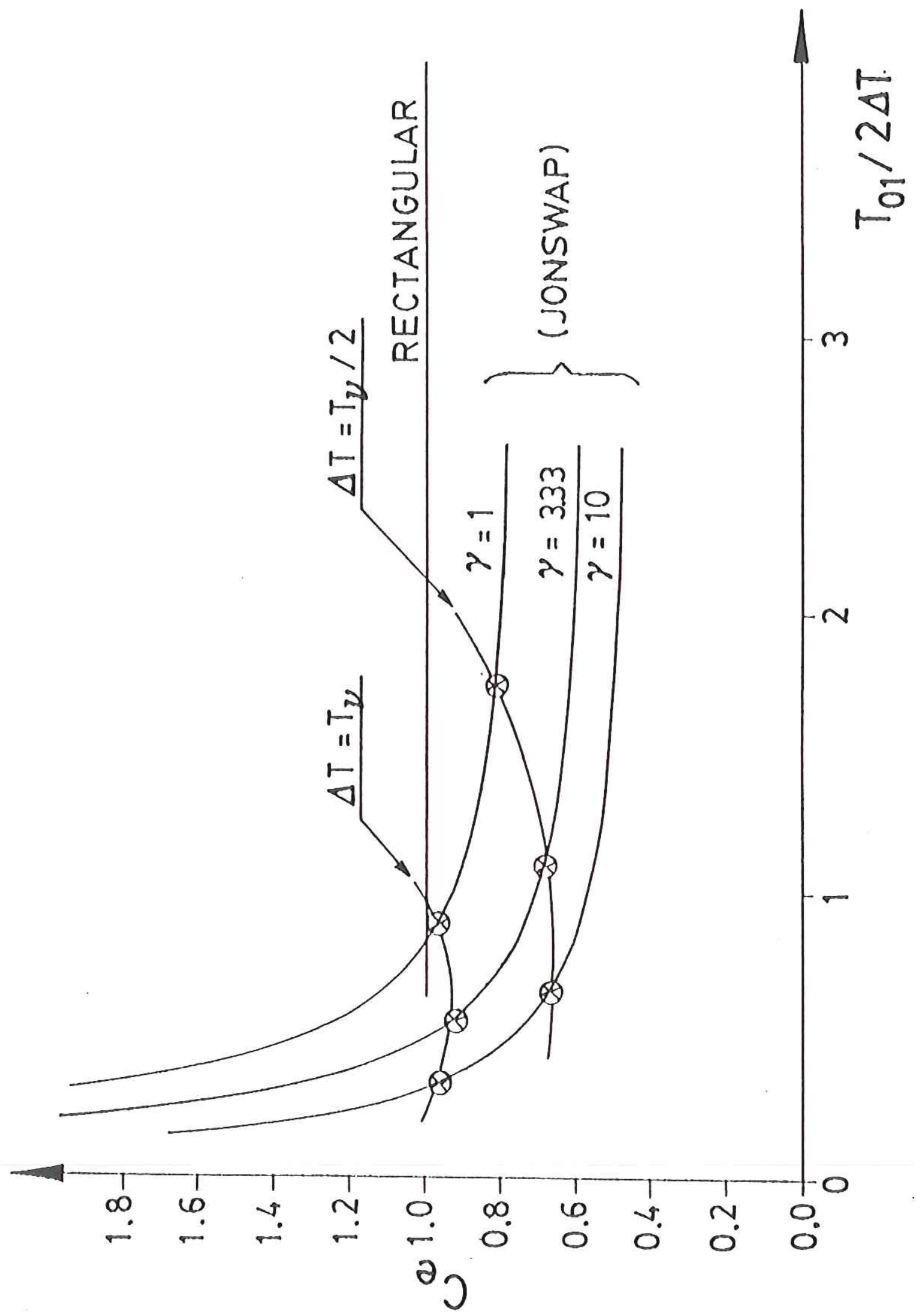

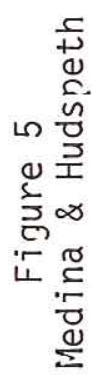




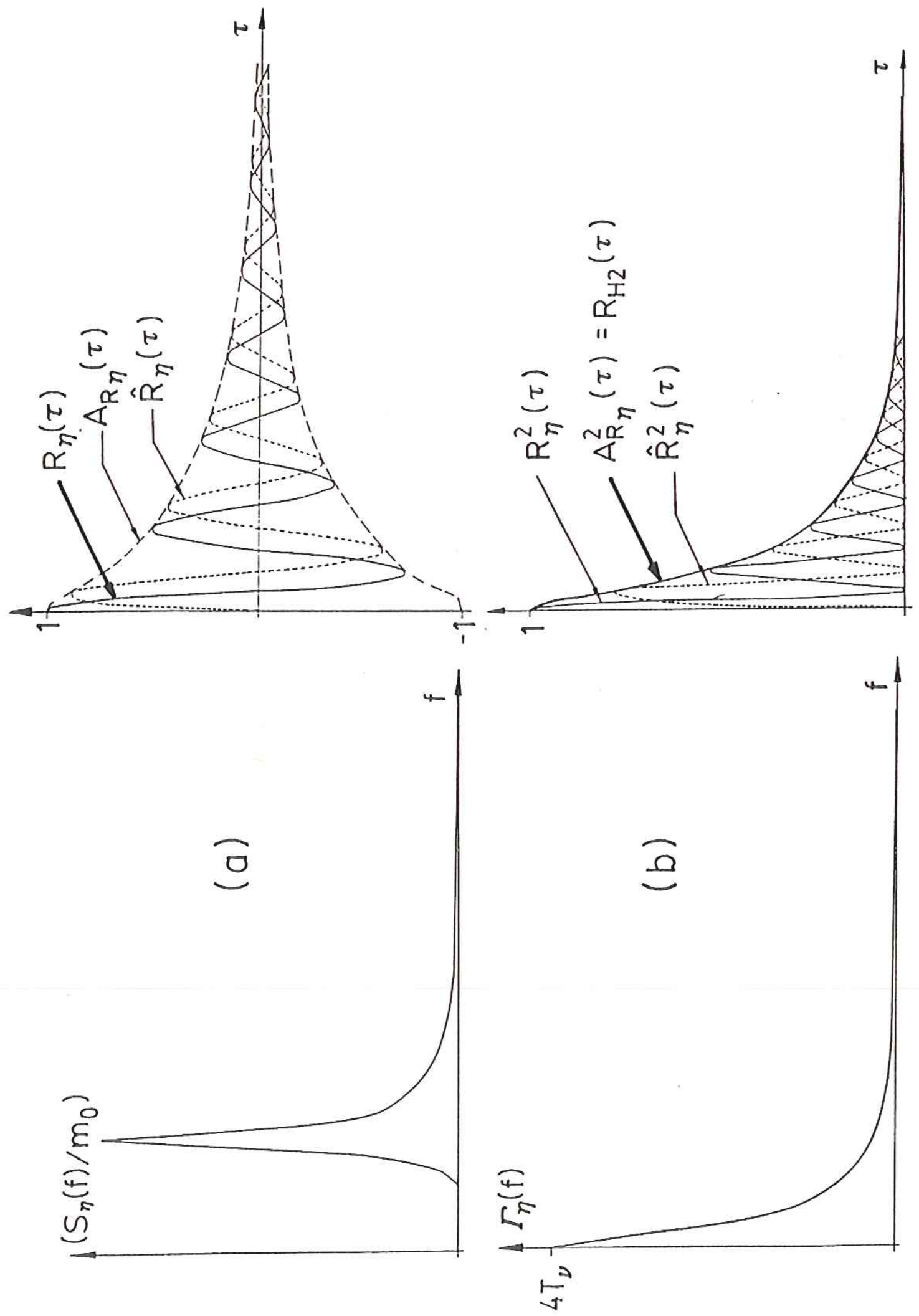

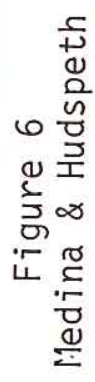




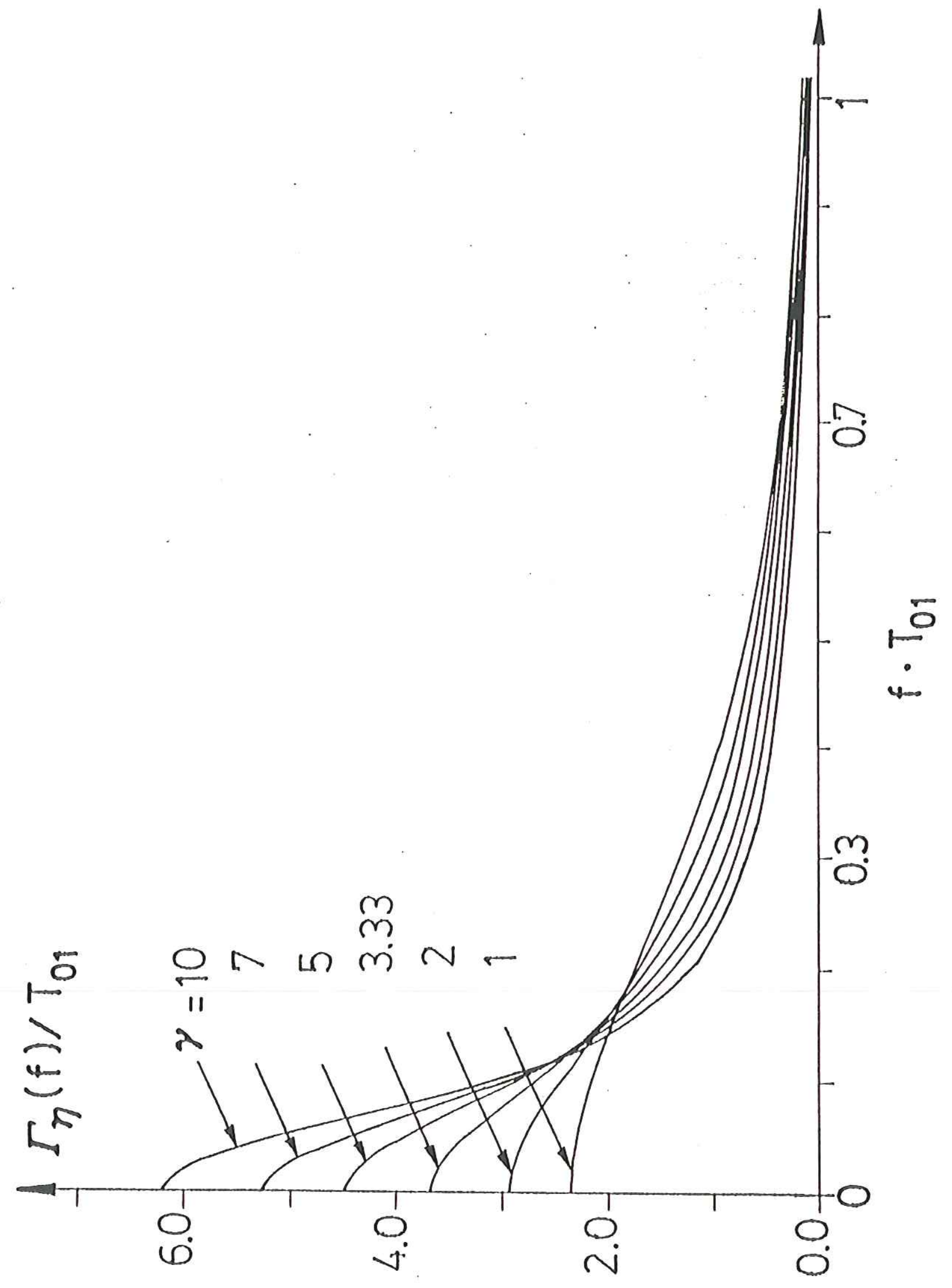




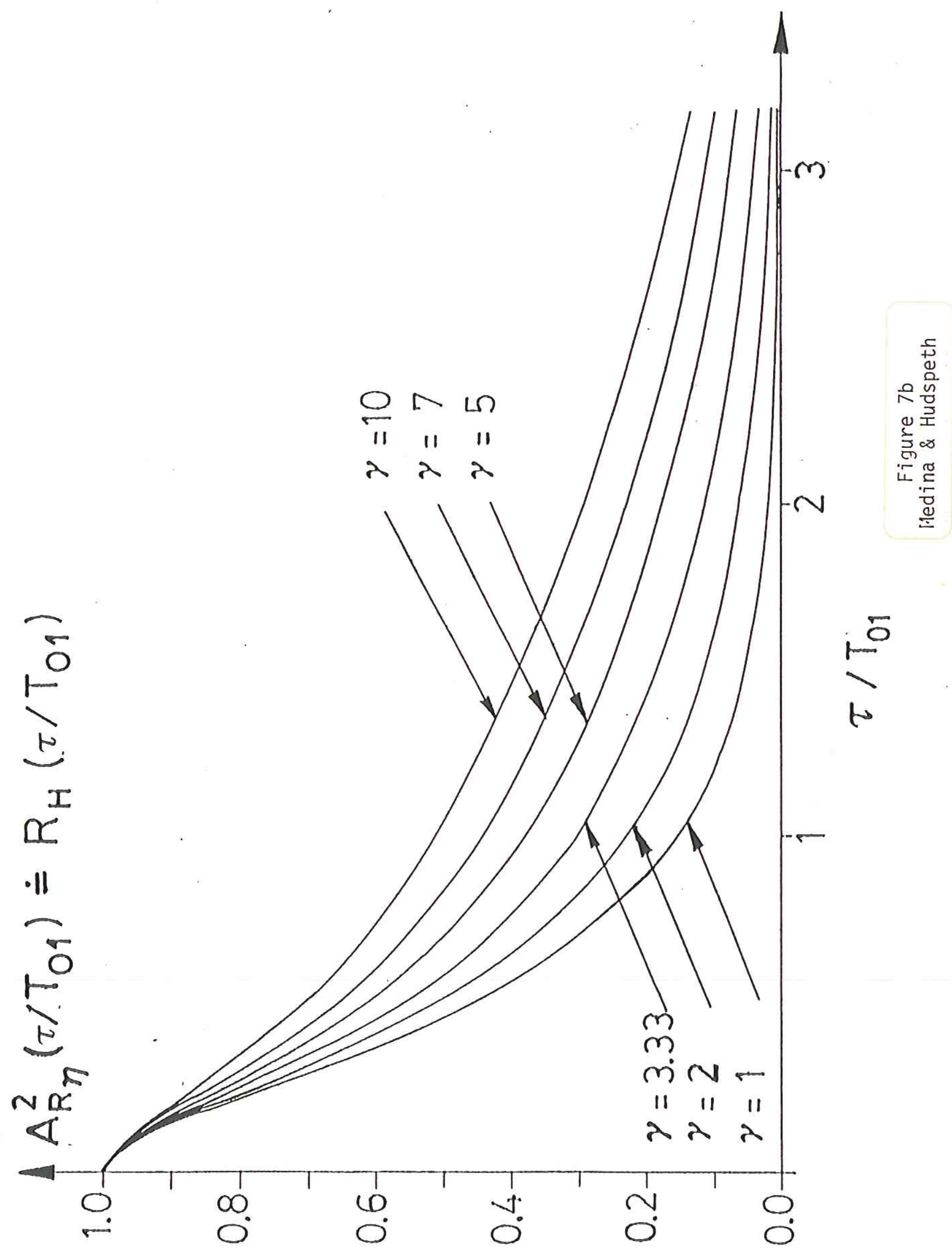




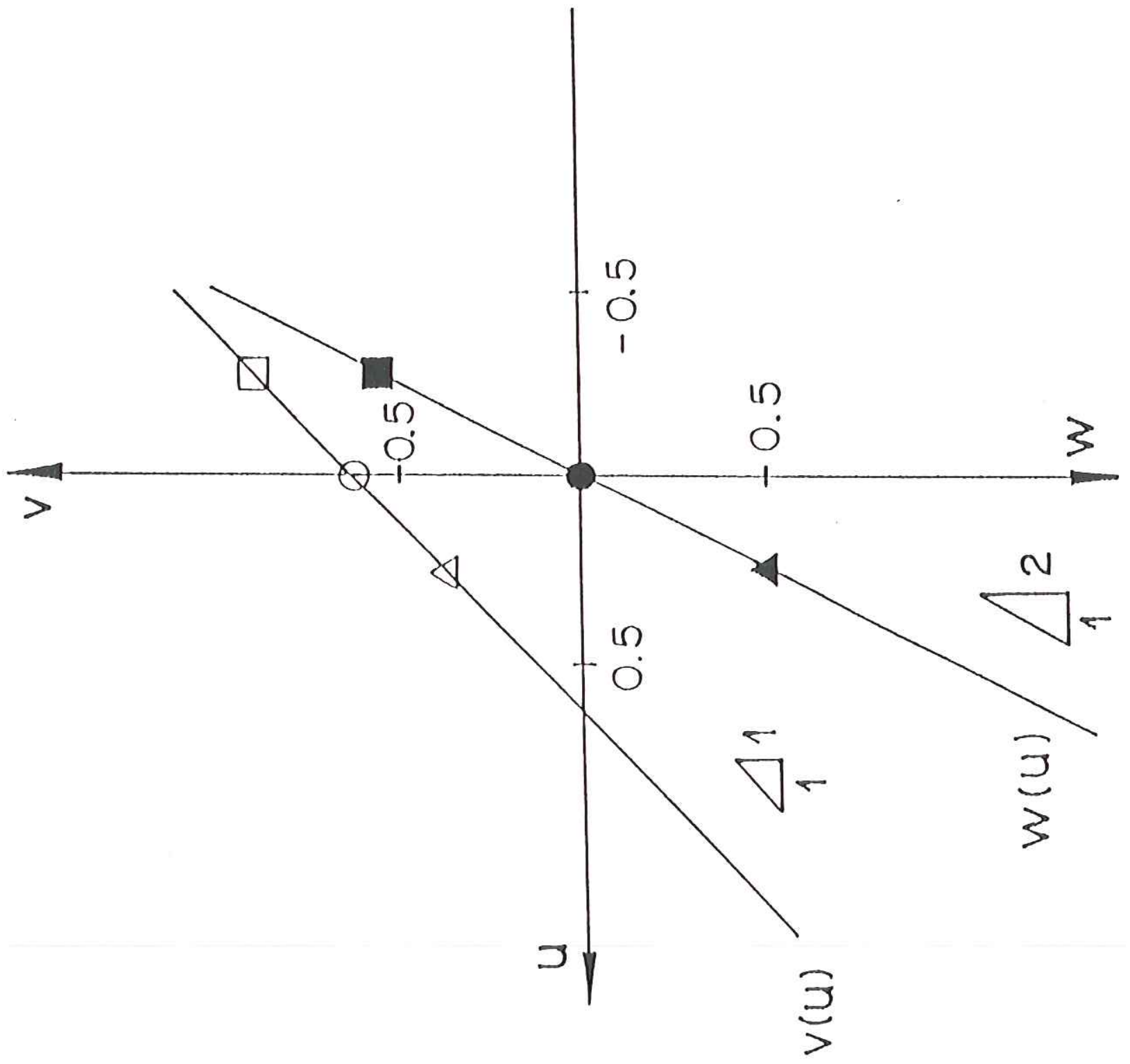

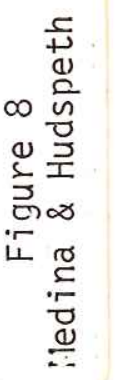




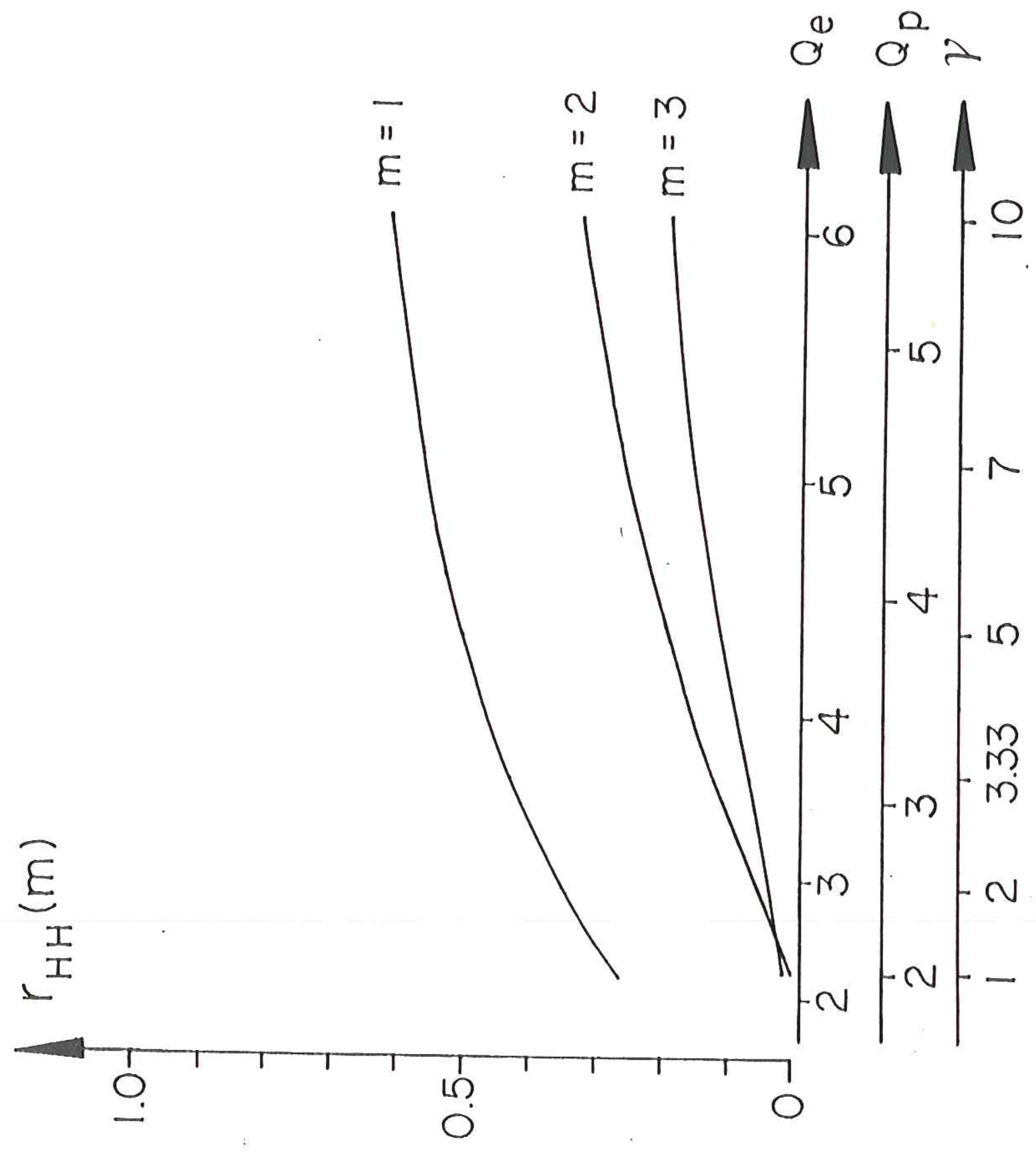




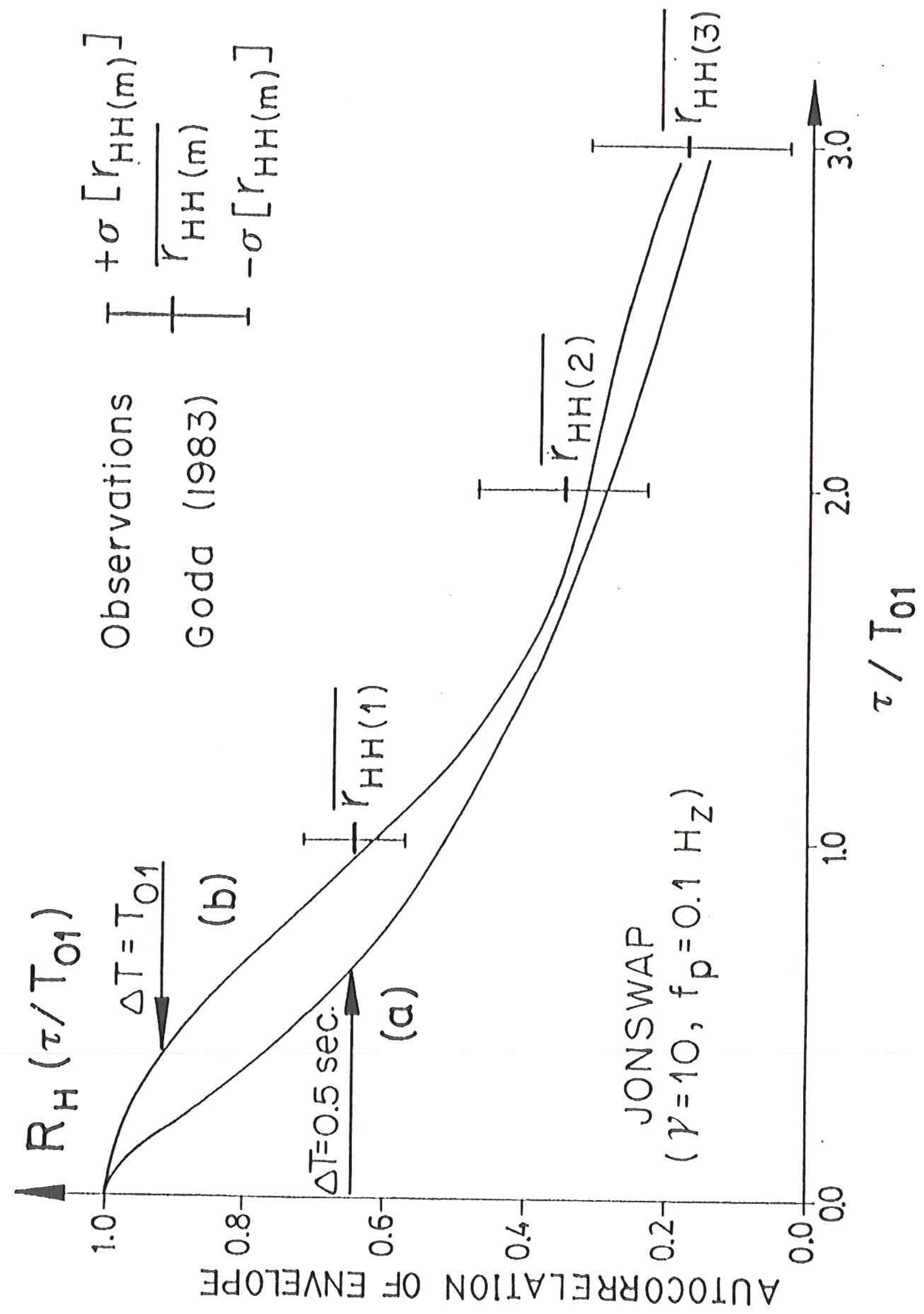




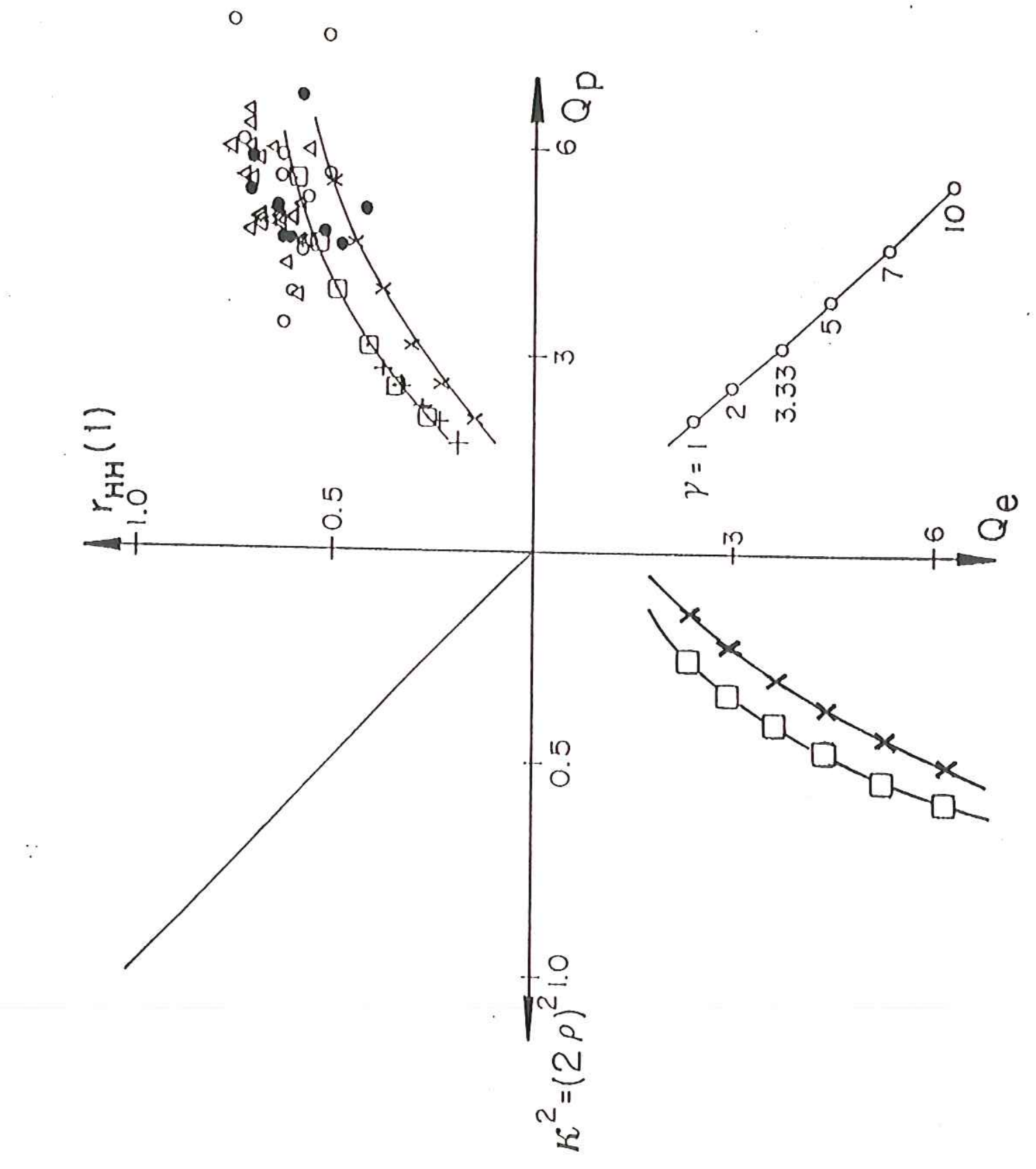

